\title{
Molar and latent models of cognitive slowing: Implications for aging, dementia, depression, development, and intelligence
}

\author{
DONALD L. FISHER and ROBERT A. GLASER \\ University of Massachusetts, Amherst, Massachusetts
}

\begin{abstract}
The time that it takes a group of participants to respond in simple cognitive tasks varies systematically with the identity of the group. For example, on most tasks, older adults take longer to respond than younger adults. Similarly, on most tasks, younger children take longer to respond than mature children. More generally, response time has been found to vary reliably with a number of other factors that differentiate groups of participants, including the levels of dementia, depression, and intelligence. For each factor, investigators have sought to determine whether the various mental processes are slowed identically as the level of impairment increases. They have based this determination largely on the relation between the overall response times of the relevant groups. Here it is shown how one can base this determination on the relation between the speeds of the individual latent or mental processes governing the performance of the target groups. Such a shift in emphasis has three important advantages: it reduces the possibility of falsely accepting or rejecting the hypothesis that all processes are slowed identically; it pinpoints the actual processes that are lengthened disproportionately when processes are not slowed identically; and it makes possible the rigorous testing of the effects of changes in speed on other dependent variables (e.g., accuracy).
\end{abstract}

The response times of one group of experimental participants are frequently found to be related simply and systematically to the response times of a second group of participants. For example, a number of investigators have found that the response times of older adults can be expressed as a simple linear function of the response times of younger adults (Birren, 1965; Cerella, Poon, \& Williams, 1980; Hale, Myerson, Faust, \& Fristoe, 1995; Salthouse \& Somberg, 1982). Similarly, at the other end of the developmental spectrum, investigators have found that the response times of younger children can be written as a linear function of the response times of more mature children (Cerella \& Hale, 1994; Hale, 1990; Kail, 1991). Simple linear relations have also been found to exist between the response times of participants differing in their level of dementia (Madden, Nebes, \& Allen, 1992;

The research was supported in part by a grant from the National Institutes of Health (NIA No. R01 AG12461) to the first author. This work is based in part on talks delivered to the Cognitive Aging Conference, Atlanta, April 1992, and to the Cognitive Aging Group, Georgia Institute of Technology, June 1992. The first author especially wants to thank Dan Fisk both for introducing him to many of the problems addressed in this article and for the many spirited discussions of these problems over one too many shared espressos in funky, out-of-the-way midwestern cafes. He also wants to thank Jerry Myers for various of the statistical tests he has suggested, and John Cerella, Sandra Hale, Chris Hertzog, Duncan Luce, Joel Myerson, James Townsend, and Michael Wenger for their comments on an earlier draft. Finally, he wants to thank Chuck Watson and the Indiana University Institute for the Study of Human Capabilities for their assistance throughout the project. Requests for reprints should be addressed to D. L. Fisher, Department of Mechanical and Industrial Engineering, 220 ELAB, University of Massachusetts, Amherst, MA 01003 (e-mail: fisher@ecs.umass.edu).
Nebes \& Brady, 1992; Nebes \& Madden, 1988), depression (Hart, Kwentus, Wade, \& Hamer, 1987; Niederehe, 1986), and intelligence (Hertzog, 1989, 1992; Vernon \& Jensen, 1984).

The fit of linear models of cognitive slowing to the results of such studies is extraordinary. The models have explained upwards of $99 \%$ of the variance in the mean response times across a wide range of different tasks. It is frequently argued that the linear slowing functions are general ones that do not vary from task to task (Birren, 1965; Cerella et al., 1980; Nebes \& Brady, 1992; Salthouse \& Somberg, 1982). Specifically, let $O_{i}$ represent the response time of the slower cohort, and let $Y_{i}$ represent the response time of the faster cohort. Then, the expected response time $E\left[O_{i}\right]$ of the slower cohort in task $i$ is written as the sum of a constant plus some multiple of the expected response time $E\left[Y_{i}\right]$ of the faster cohort in task $i$ :

$$
E\left[O_{i}\right]=\alpha+\beta E\left[Y_{i}\right]
$$

where the distribution of the response time $Y_{i}$ of the faster cohort (but neither parameter of the regression; i.e., neither $\alpha$ nor $\beta$ ) varies with the task. This linear model of general slowing (Equation 1) will be referred to here as a molar model, because the overall response times of the slower cohort are a function of the overall response times of the faster cohort (and not the durations of the individual underlying processes).

Sometimes it is argued that the molar slowing functions are common to a specific domain $d$ (e.g., the lexical domain), but that they vary across domains (Hale \& Myerson, 1992). In the extreme, it has been argued that the molar slowing function varies from one task to the 
next even within domains (Fisk \& Rogers, 1991; Fisk, Fisher, \& Rogers, 1992). Thus, one can write

$$
E\left[O_{i}\right]=\alpha_{i}+\beta_{i} E\left[Y_{i}\right],
$$

where $\alpha_{i}$ and $\beta_{i}$ are the task-specific slowing factors. This model (Equation 2) will be referred to here as a molar linear model of task-specific slowing.

More complex molar models have been considered. These more complex models include power functions (Hale, Myerson, \& Wagstaff, 1987; Myerson, Hale, Wagstaff, Poon, \& Smith, 1990) and probability mixtures of linear functions (Cerella, 1985). However, the more complex models are not typically needed when the response times are in the range $0-2,000 \mathrm{msec}$, the range of response times found in many (though not all) experiments (Cerella \& Hale, 1994).

\section{Latent Models: False Positive and False Negative Decisions}

Interest centers not only on the slowing of the molar (overall and observable) response times of one cohort relative to some other baseline cohort, but also on the slowing of the latent (underlying and not observable) processes of the two cohorts (these terms and others are defined in Table 1). As noted above, Equations 1 and 2 are examples of molar models. Examples of latent models can be similarly constructed. We will develop two such models, a latent model of common slowing and a latent model of process-specific slowing. To begin, it is necessary to assume something about the architecture of cognition in both the faster and the slower cohorts. To keep things simple, assume that the processes $x_{1}, \ldots, x_{n}$ are arranged in series in both cohorts. Let $X_{j}$ represent the duration of process $x_{j}$ in the faster cohort. Then, the expected time $E[Y]$ that it takes the faster cohort to complete all $n$ processes can be written as the sum of the expected durations of each of the processes:

$$
E[Y]=E\left[X_{1}\right]+E\left[X_{2}\right]+\ldots+E\left[X_{n}\right] .
$$

We can now compute the response times of the slower cohort. To begin, consider this computation when the latent model is one of common slowing. Specifically, in a latent model of common slowing, we will assume that all processes are slowed identically. An example can make clear most quickly what we mean. To keep the example simple, assume that all processes are slowed proportionately-say, by some factor $\beta$ (obviously other slowing functions are possible). Then, the expected time $E[O]$ that it takes the slower cohort to complete all processes is obtained from the latent model for the faster cohort (Equation 3) by multiplying the expected duration of each latent process by the common slowing factor $\beta$ :

$$
E[O]=\beta E\left[X_{1}\right]+\beta E\left[X_{2}\right]+\ldots+\beta E\left[X_{n}\right] .
$$

Next, consider the computations when the latent model is one of process-specific slowing. In such a model, we will assume that the processes are not slowed identically. So, for example, we might assume that each process $x_{j}$ is slowed by a different factor $\beta_{j}$, so that:

$$
E[O]=\beta_{1} E\left[X_{1}\right]+\beta_{2} E\left[X_{2}\right]+\ldots+\beta_{n} E\left[X_{n}\right] .
$$

Note that the preceding latent models of common and process-specific slowing are both referred to as multiplicative models, because the slowing is proportional.

False positive decisions. Typically, the durations of the latent processes (and therefore the associated latent slowing functions) are not estimated directly. Rather, the form of the latent slowing function is inferred by investigators from the form of the molar slowing function. Specifically, it is assumed that if the molar slowing function is a general one (i.e., does not vary from one task or domain to the next), the latent slowing function is a common one (i.e., does not vary from one process to the next). For example, suppose that the response times of one group are slowed proportionately by some factor $\beta$ across several different tasks. Then, it would be inferred that each of the latent processes is slowed proportionately by the same factor $\beta$. Examples in the literature of just this inference have been common in the past, but are now becoming less so. Specifically, it is now recognized that the inference that the latent slowing function is a common one if the molar slowing function is a general one runs into both logical and statistical roadblocks (Fisk \& Fisher, 1994; Fisk et al., 1992).

Logically, it can be shown that if the form of the molar slowing function is a general one, each of the latent processes need not be slowed identically (Fisk \& Fisher, 1994; Hale et al., 1995). An example can quickly indicate the problem. Note that in Figure 1a the response times of the slower cohort are twice as long as the response times of

Table 1

Terms

Molar slowing function

Molar model of general slowing

Molar model of task-specific slowing

Latent slowing function (no resource constraints)

Latent slowing function (resource constraints)

Latent model of common slowing

Latent model of process-specific slowing

Endogenous process

Exogenous process
A function that relates the expected response times of two cohorts

A molar slowing function that does not vary across tasks

A molar slowing function that does vary across tasks

A function that relates the durations of a latent process that appears in the networks of two cohorts

A function that relates the work rates of a latent process that appears in the networks of two cohorts

A latent slowing function that does not vary across processes

A latent slowing function that does vary across processes

Any psychological process

A process external to a participant that influences response times 
the faster cohort in each of Tasks 1 ( $300 \mathrm{msec}$ for the younger adults, $600 \mathrm{msec}$ for the older adults), 2 (600 vs. $1,200 \mathrm{msec}$ ), and 3 (900 vs. $1,800 \mathrm{msec}$ ). Clearly such a finding is consistent with a molar model of general slowing. Specifically, set $\alpha=0$ and $\beta=2$ in Equation 1. Surprisingly, the same finding is also consistent with a latent model of process-specific slowing. For example, in Figure $1 \mathrm{~b}$, it is assumed that each task consists of two latent processes arranged in series. Furthermore, it is assumed that the duration of the first process is increased by a factor of 5 (e.g., in Task 1, the duration of the first process is increased from $100 \mathrm{msec}$ in the faster cohort to $500 \mathrm{msec}$ in the slower cohort), whereas the duration of the second process is decreased by a factor of 0.5 (e.g., in Task 1 , the duration of the second process is decreased from 200 to $100 \mathrm{msec}$ ). Specifically, set $E\left[X_{1}\right]=100, E\left[X_{2}\right]=200$, $\beta_{1}=5$ and $\beta_{2}=.5$ in Equation 5 . The important thing to note here is that the two models, the molar model of general slowing (Figure 1a) and the latent model of process-

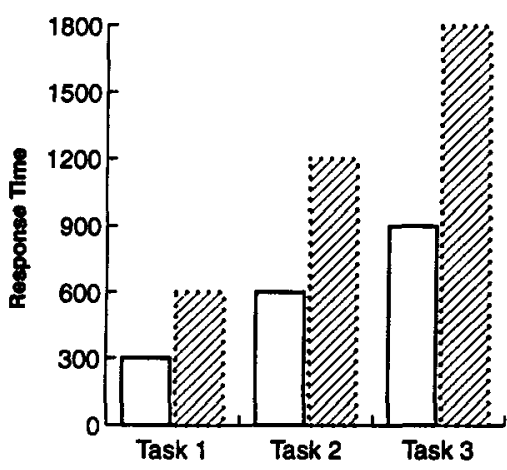

(a) Molar Model of Conoral Slowing

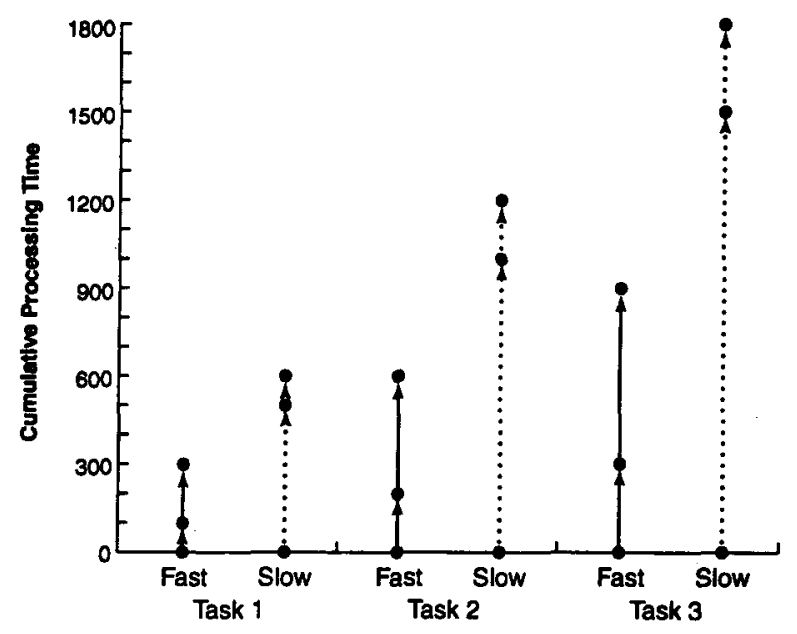

(b) Latent Model of Process-Specific Slowing

Figure 1. False positive evaluations of the molar model of general slowing. The response times in Tasks 1, 2, and 3 are identical for (a) the molar model of general slowing and (b) the latent model of process-specific slowing. Solid arrow lengths represent duration of younger adults' latent processes; dotted arrow lengths represent duration of older adults' latent processes. specific slowing (Figure 1b), each predict the same absolute response times in Tasks 1,2, and 3 even though the latent processes are slowed by very different factors. Thus, an investigator relying solely on the knowledge that the form of the molar slowing function was a general one could falsely accept the latent model of common slowing (this will be called a false positive decision).

Statistically, it can be shown that even if the best fit of the molar model of general slowing is extraordinarily good, the latent slowing function for a particular process can vary radically from one task to the next (Fisk \& Fisher, 1994; Fisk et al., 1992; Molenaar \& van der Molen, 1994; Perfect, 1994). An example can quickly indicate the problem. Specifically, consider the hypothetical cross plot in Figure 2a. Each point represents two means, the means of the slower and faster cohorts in a particular condition. Six means were computed in the first task (plotted as the three points at the lower left) and six in the second (plotted as the three points in the upper right). A molar model of general slowing (in this case a simple linear slowing function) explains over $95 \%$ of the variance. Thus, it would appear that the molar model of general slowing is the correct one. However, note that in the first task, the response times of the slower cohort (say, older adults in this case) decrease with increases in the response times of the faster cohort (say, younger adults), whereas in the second task, the response times of the slower cohort increase with increases in the response times of the faster cohort. Thus, the correct molar model cannot be a general one. In summary, an investigator relying solely on the good fit of the molar model of general slowing to response times as represented in a cross plot (i.e., a Brinley plot; Brinley, 1965) could falsely accept the molar model of general slowing (and therefore falsely accept the latent model of common slowing).

False negative decisions. Just as it is typically assumed that if the molar slowing function is a general one, the latent slowing function is a common one, so, conversely, it is assumed that if the molar slowing function is not a general one (i.e., is task specific), the latent slowing functions are not common ones (i.e., are process specific). Until recently, the latter assumption was rarely mentioned, simply because the molar slowing function was assumed to be a general one, at least within and most often across domains. However, recently, a number of investigators have argued that the molar slowing function is not necessarily a general one (Fisher, in press; Fisher, Fisk, \& Duffy, 1995; Fisk \& Fisher, 1994; Fisk et al., 1992; Kleigl, 1992; Kleigl, Mayr, \& Krampe, 1994; Laver \& Burke, 1993; Mayr \& Kleigl, 1993). Thus, the assumption above may come to play an increasingly important role. In this case, it is important to recognize that the inference that the latent slowing function is process-specific if the molar slowing function is task-specific runs into troublesome logical and statistical roadblocks similar to those described above (Fisk \& Fisher, 1994; Fisk et al., 1992).

Logically, it can be shown that if the form of the molar slowing function is task-specific, each of the latent cognitive processes could be slowed identically. Again, an 
(a)

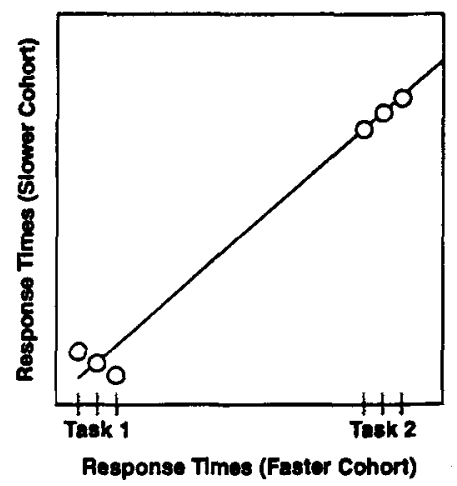

(b)

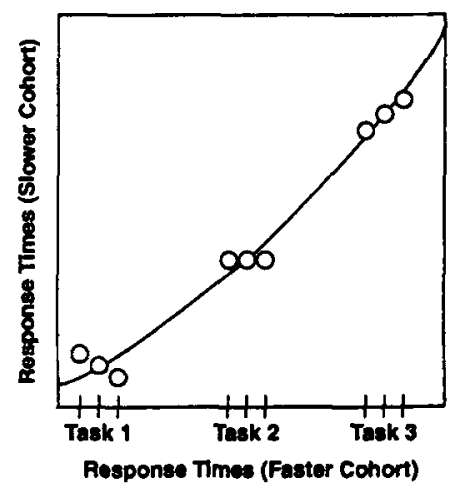

Figure 2. A cross plot of older against younger adults' response times. (a) A molar linear model of general slowing is fit to the data. (b) A molar power model of general slowing is fit to the data.

example can quickly indicate the problem, although it must necessarily be slightly more complex than the previous example. Assume that there are three tasks and the response times of the faster and slower cohort are known for each task (Figure 3a). Note that the response times of the slower cohort are not a constant multiple of the response times of the faster cohort across tasks but instead vary from task to task. Specifically, set $\alpha_{1}=\alpha_{2}=\alpha_{3}=0$, $\beta_{1}=224 / 168, \beta_{2}=240 / 168$, and $\beta_{3}=252 / 168$ in Equation 2 . Then, the results are consistent with a molar model of task-specific slowing.

Now, consider a latent model of processing in this task. To begin, we want to assume that, for both cohorts, each task requires the completion of three processes (Figure $3 b$ ). The initial two processes are executed in parallel. Both must be completed before the final process can begin. The participant responds when the final process has been completed. So, for example, consider the network displayed in Figure 3b, which governs the performance of the faster cohort in Task 1 . The first two processes take 100 and 400 time units, indicated by the solid and dashed lines, respectively. Since the third process cannot be completed until both of the first two processes have been completed, it does not begin until 400 time units have elapsed. Thus, the response time is 600 time units. Next, we want to assume that only one of the two initial parallel processes (the process drawn as a solid line for the faster cohort and as a dotted line for the slower co- hort) represents a latent cognitive (endogenous) event. The duration of an endogenous process is influenced by the factor that differentiates cohorts (e.g., age). Finally, we want to assume that the second of the two initial processes (the process drawn as a dashed line for both cohorts) represents a latent environmental (exogenous) event. For example, the other process might represent the interval between the presentation of stimuli in a priming experiment (Laver \& Burke, 1993) or a double stimulation experiment (Pashler \& Johnston, 1989). The significance of the presence of an exogenous process is that its duration is not influenced by the factor that differentiates cohorts. Rather, the duration of such a process is determined entirely by the experimenter or the environment.

The time that it takes a participant to respond when a task contains both endogenous and exogenous processes is a complex function of the durations of both sets of processes (Schweickert, Fisher, \& Goldstein, 1993). However, we are interested in the slowing of just the set of endogenous processes. Specifically, in our example, we are

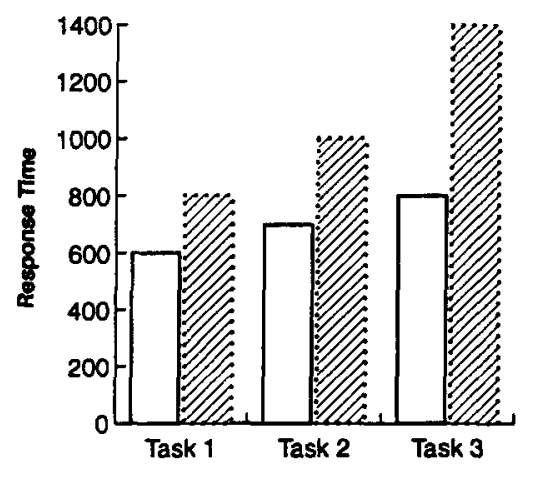

(a) Molar Model of Task-Specltic Slowing

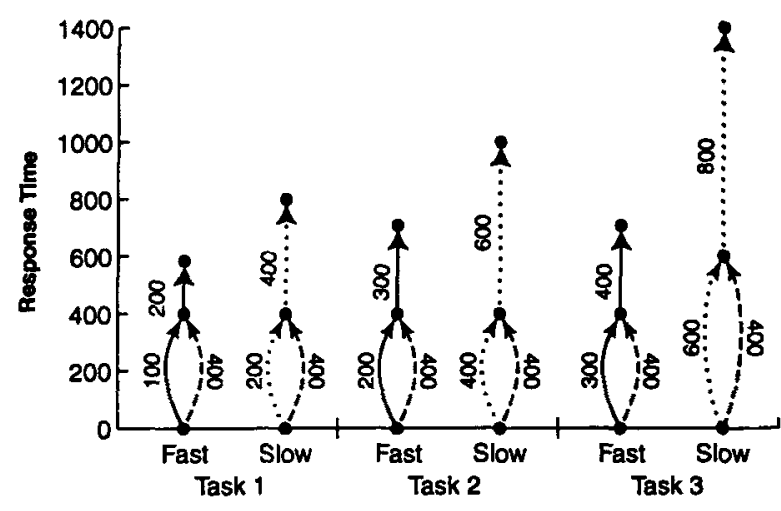

(b) Latent Model of Common Slowing

Figure 3. False negative evaluations of the molar model of general slowing. The response times in Tasks 1, 2, and 3 are identical for the example models in panels a and $b$. Solid arrows represent younger adults' latent processes; dotted arrows represent older adults' latent processes; dashed arrows represent exogenous processes. 
interested in the slowing of just the two endogenous processes. Careful inspection of Figure $3 \mathrm{~b}$ indicates that the durations of the latent endogenous processes of the slower cohort (the dotted lines) are a constant multiple (2) of durations of the latent endogenous processes of the faster cohort (the solid lines) in each of the three tasks. Thus, the latent model of common slowing is the correct one (since all cognitive or endogenous processes are slowed identically), even though the molar model is task specific. It follows that an investigator relying solely on knowledge that the form of the molar slowing function was task specific could falsely accept the latent model of process-specific slowing (this will be called a false negative decision).

Statistically, it can be shown that even if the best fitting model of general slowing is a task-specific one, the latent slowing function can be a common one. The conclusion follows directly from the preceding logical analysis. Specifically, if there are exogenous processes that determine how long it takes participants to perform a task, a molar model of task-specific slowing will necessarily fit better than a molar model of general slowing, even when all of the endogenous processes are slowed identically. Thus, on the basis of the good fit of a molar model of task-specific slowing, one could reject a molar model of general slowing (and therefore falsely reject a latent model of common slowing).

Ideally, one would like to avoid false positive and false negative decisions, both logical and statistical. Here, it is argued that in order to avoid logically incorrect inferences, it is necessary to specify the general characteristics of the latent model that governs performance in each task. In particular, it is necessary at a minimum to identify for each task the type of architecture, the limits on resources, and the possible dependencies that exist among the various process durations. Similarly, in order to avoid statistically incorrect inferences, it is argued that it is necessary not only to know the general characteristics of the latent model described above, but also actually to fit such models to each of the tasks included in a meta-analysis (or each of the conditions modeled in a single experiment).

\section{Latent Models: Process Durations}

A focus on latent models is useful not only because it can help reduce the incidence of false positive and false negative decisions. A focus on such models can also be used to identify the slowing of individual latent processes. Knowledge of just how much each process is slowed has three potential advantages. First, such knowledge can be used to determine whether the slowing of a particular process is more than would be expected just on the basis of knowledge of the average slowing of the individuals in the relevant cohort. Methods for identifying the processes that are slowed more than would be expected are now available (Madden, Pierce, \& Allen, 1992). Such methods work well when the processes are all arranged in series. However, the current methods do not extend to tasks in which the processes that govern performance assume a more complex architecture. This restriction does not apply to the latent model methodology described herein. Specifically, the latent models can be used to identify the seriously compromised processes in networks with both serial and parallel processes.

Second, knowledge of which processes are and are not slowed can potentially be used to test more detailed hypotheses about the role that a given neuroanatomical structure has on cognitive slowing. For example, a better understanding of event-related brain potentials may soon make it possible to link a specific cognitive process to a specific neuroanatomical structure (Bashore, 1993). It would then become possible to understand much better the relation between neuroanatomy and the speed of cognition. In particular, given current neural models of slowing (e.g., those of Cerella, 1990; Cerella \& Hale, 1994), all processes that are mediated by a given structure should presumably yield the same slowing in a particular cohort. Evidence that this was not the case would require much more complex neural models of slowing than have been considered to date.

Finally, knowledge of which processes are and are not slowed can potentially lead to real improvements in the lives of cohorts with a marked speed deficit. For example, it is well known that older adults are overinvolved in accidents at signalized left-turn intersections (Hanowski, Bittner, Knipling, Byrne, \& Parasuraman, 1995; Staplin \& Fisk, 1991). Some investigators have hypothesized that it is a shrinking of the useful field of view that explains older adults' overinvolvement in these accidents (Ball \& Owsley, 1991). However, it is not known which of the various cognitive and perceptual processes that determine the size of the useful field of view are responsible for the problems that the older adults experience at a signalized left-turn intersection. Knowledge of the slowing of each of the latent processes is a necessary first step in any remediation effort (Fisher, 1993).

\section{Latent Models: Speed-Related Influences}

A number of investigators have argued that increases in the time that it takes a given cohort to process information may affect their performance not only on speeded tasks, but also on a number of other tasks in which speed is not so obviously a factor (Hertzog, 1989; Salthouse, 1991; Salthouse, Kausler, \& Saults, 1988; Salthouse \& Mitchell, 1990; Schaie, 1989). For example, Salthouse (1996) finds that basic measures of processing speed predict much of the variability in the performance of older adults on tests of memory for paired associates. Ideally, one would like to verify that the slowing of a given cognitive process in a memorial task by a particular proportion did indeed lead to the observed memory deficit. The quantitative link between processing speed and memory deficit can then be fashioned with a latent model. Latent models sufficient for this task have recently been developed (Fisher, in press; Fisher, Wisher, \& Ranney, 1996). 
More generally, a quantitative link between processing speed and other dependent variables can be forged only when the relevant latent model is detailed.

\section{PERT NETWORKS}

In order to understand the relation between the latent and molar slowing functions and thereby avoid false positive and false negative decisions, it is necessary to have some model of participants' performance. In simple cognitive tasks, this performance is frequently modeled as a network of mental processes. A number of different network architectures have been proposed. These include PERT networks (Schweickert, 1978), associative networks (e.g., Anderson \& Bower, 1974), connectionist networks (McClelland, Rumelhart, \& the PDP Research Group, 1986; Rumelhart, McClelland, \& the PDP Research Group, 1986), and queuing networks (e.g., Fisher, 1982; Liu, 1996; Miller, 1993; Rouse, 1980). More recently, Fisher (1985) has shown that many stochastic networks are special cases of order-of-processing diagrams. The significance of such diagrams is that it is known both how to compute the moments of the response time (Fisher \& Goldstein, 1983; Goldstein \& Fisher, 1991), and, in many cases, how to test specific hypotheses about the structure of the underlying diagram (Schweickert, 1978; Schweickert \& Townsend, 1989). In order to keep the discussion relatively free of technical details, attention will be confined at this point to PERT networks (a more general discussion can be found in Schweickert et al., 1993).

Briefly, a PERT network $G(V, E)$ is a directed, acyclic graph that consists of a set of vertices (nodes) $V$ and a set of edges (arcs) $E$ with a single source and a single sink. The nodes represent AND gates. The $n \operatorname{arcs} x_{1}, \ldots, x_{n}$ represent processes. For example, consider the PERT network in Figure 4 . The nodes are drawn as filled circles. The source is labeled $o$; the sink, $r$ (there is no need to label the interior nodes, and thus no labels are given). The arcs are drawn as arrows connecting pairs of nodes. No process represented by an arc can begin executing until all its predecessors have completed executing. For example, process $x_{5}$ cannot begin executing until both processes $x_{3}$ and $x_{4}$ have completed executing. All processes that can begin do so immediately. For example, both processes $x_{2}$ and $x_{3}$ can and therefore do begin immediately after $x_{1}$ finishes.

Consider now the faster cohort. The time $Y$ that it takes the faster cohort to respond is equal to the time that it

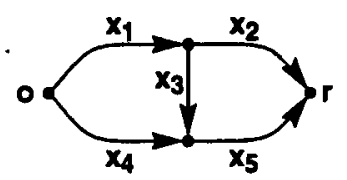

Figure 4. An (incomplete) Wheatstone bridge PERT network. The source is represented as $o$; the sink, as $r$. The processes are labeled $x_{1}, \ldots, x_{5}$.) takes to complete the last finishing process. $Y$ is typically written as a function of the durations of the paths in the network. A path is defined in this context as a sequence of processes (arcs) from the source to the sink (e.g., the processes $x_{1}, x_{3}$, and $x_{5}$ form a path). Let $X_{h_{k}}$ represent the duration of the $k$ th process on path $h$. Then, the duration $A_{h}$ of a path $h$ with processes $x_{h_{1}}, x_{h_{2}}, \ldots, x_{h_{n(h)}}$ is defined as the sum

$$
A_{h}=X_{h_{1}}+X_{h_{2}}+\ldots+X_{h_{(h)}},
$$

where $n(h)$ is the number of processes on path $h$. Set $p$ equal to the number of paths linking the start and finish nodes. Then, the response time $Y$ can be shown to equal the duration of the longest of the $p$ paths. For example, in the simple network in Figure 4,

$$
Y=\max \left\{X_{1}+X_{2}, X_{1}+X_{3}+X_{5}, X_{4}+X_{5}\right\} \text {. }
$$

Consider next the slower cohort. The model of the slower cohort's response times can be either a molar or a latent one. Definitions of these models are necessarily more complex when the underlying architecture is not the simple serial one described in the introduction. As above, let $X_{j}$ represent the duration of process $x_{j}$ when the faster group is performing a particular task. Let $\Psi_{j}$ be the duration of process $x_{j}$ when the slower group is performing the same task (it is assumed here that the two groups process information identically, so that the same label, $x_{j}$, can be used to identify processes, although different labels, $X_{j}$ and $\Psi_{j}$, are needed for the process durations). Then, in the molar model, the expected response time $E[O]$ of the slower group is assumed to be written as a function of the expected response time $E[Y]$ of the faster group:

$$
E[O]=f(E[Y]),
$$

where $f$ is the molar slowing function. (Note that Equation 8 is a more general statement of Equations 1 and 2.) In the related latent model, the expected response time $E[O]$ of the slower group is written as a function of the duration of the processes governing the slower groups' performance. The duration $\Psi_{j}$ of each of these processes is, in turn, written as a function $f_{j}\left(X_{j}\right)$ of the duration of the corresponding processes governing the faster groups' performance:

$$
\begin{aligned}
E[O]= & E\left[\max \left\{\Psi_{1_{1}}+\ldots+\Psi_{1_{n(1)}}, \ldots, \Psi_{p_{1}}+\ldots+\Psi_{p_{n(p)}}\right\}\right] \\
= & E\left[\operatorname { m a x } \left\{f_{1_{1}}\left(X_{1_{1}}\right)+\ldots+f_{1_{n(1)}}\left(X_{\left.1_{n(1)}\right)}\right), \ldots,\right.\right. \\
& \left.\left.f_{p_{1}}\left(X_{p_{1}}\right)+\ldots+f_{p_{n(p)}}\left(X_{p_{n(p)}}\right)\right\}\right],
\end{aligned}
$$

where $f_{h_{k}}$ is the latent slowing function for, and $\Psi_{h_{k}}$ the duration of, the $k$ th process $x_{h_{k}}$ that is completed along the $h$ th path in the PERT network governing the slower cohorts' behavior. (Note that Equation 9 is a more general statement of Equations 4 and 5.) Thus, in a very simple case, one might write $\Psi_{j}=f_{j}\left(X_{j}\right)=\beta X_{j}$ for $j=1, \ldots, n$. (The notation used in this section and more generally the notation used throughout the article to describe PERT networks is summarized in Table 2.) 


\section{LOGICAL ANALYSES: FALSE NEGATIVE DECISIONS}

Given that a PERT network governs participants' behavior, it is now possible to identify the set of circumstances that must obtain if one is to avoid the false negative decisions described above. Specifically, it is shown under what circumstances it is possible to infer that the molar slowing function is a general one when the latent slowing function is known to be a common one. It then follows from the contrapositive that as long as these circumstances obtain, if the molar slowing function is known not to be a general one, it can be inferred that the latent slowing function is not a common one. Thus, one can avoid rejecting incorrectly a latent model of common slowing (i.e., avoid making false negative decisions). The set of circumstances that may lead to false positive decisions will be described in the next section.

Up to this point, it has been possible to speak in mostly generic terms, not mentioning the particular slowing factor or function under consideration. At this point, it will be useful to ground the discussion by identifying a particular slowing factor. The obvious slowing factor to choose is that of aging, since most of the research comparing the speeded responses of groups of participants has compared older and younger adults. Yet although this

Table 2

Major Notation

\begin{tabular}{|c|c|}
\hline$d$ & Index of a domain \\
\hline$h$ & Index of a path in a PERT network or OP diagram \\
\hline$i$ & Index of a task \\
\hline$j$ & Index of a process \\
\hline$k$ & $\begin{array}{l}\text { Index of the position of a process (state) along a path } h \text { in } \\
\text { PERT network (OP diagram) }\end{array}$ \\
\hline$m$ & Number of tasks \\
\hline$n$ & Number of processes in a PERT network \\
\hline$n(h)$ & $\begin{array}{l}\text { Number of processes (states) on path } h \text { in a PERT network } \\
\text { (OP diagram) }\end{array}$ \\
\hline$o$ & Source node in a PERT network \\
\hline$p$ & Number of paths in a PERT network or OP diagram \\
\hline$r$ & Sink node in a PERT network \\
\hline$s_{h_{k}}$ & $k$ th state along the $h$ th path in the $\mathrm{OP}$ diagram \\
\hline$x^{n k}$ & Latent process \\
\hline$A$ & Duration of a path in a PERT network or OP diagram \\
\hline$B$ & Random proportional slowing factor \\
\hline$G(V, E)$ & PERT network with a set $V$ of vertices and $E$ of edges \\
\hline $\mathbf{N}$ & Set of latent processes \\
\hline$O$ & Response time of slower (older) adults \\
\hline$T$ & Time that it takes to respond \\
\hline$T_{h_{k}}$ & Duration of the $k$ th state along the $h$ th path in the OP diagram \\
\hline$W^{*}$ & Work required to complete a process \\
\hline $\mathbf{W}$ & Column vector of work requirements $\left(W_{1}, \ldots, W_{n}\right)^{\prime}$ \\
\hline$X$ & Duration of a latent process (faster or younger cohort) \\
\hline $\mathbf{X}$ & Column vector of process durations $\left(X_{1}, \ldots, X_{n}\right)^{\prime}$ \\
\hline$Y$ & Response time of faster (younger) adults \\
\hline$\alpha$ & Additive constant in a molar linear model of general slowing \\
\hline$\beta$ & Proportional slowing factor \\
\hline & Expected duration of process $x_{j}$ in task $i$ \\
\hline$v(j, k, h)$ & $\begin{array}{l}\text { Rate at which work proceeds on the } j \text { th process in the } k \text { th } \\
\text { state along path } h\end{array}$ \\
\hline$\Lambda$ & $m \times n$ matrix $\left[\mu_{i j}\right]$ of process durations \\
\hline$\Psi$ & Duration of a latent process (slower or older cohort) \\
\hline
\end{tabular}

discussion will draw heavily from the aging literature, the conclusions reached about the relations between the molar and latent slowing functions will be general, and this generality will be emphasized at several points in the discussion.

It has also been possible up to this point to leave the form of the latent slowing function unspecified, not settling on an additive, multiplicative, or full linear model. Again, it will be useful to ground the discussion. Perhaps the most obvious latent slowing function to choose is a multiplicative one, since the identical molar slowing function appears to account for the vast majority of the data (Cerella \& Hale, 1994; Cerella et al., 1980). Although it is true that the estimates of the intercept of the molar linear slowing function are not always zero (Cerella, 1985), this can be shown to be consistent with separate latent multiplicative slowing functions for peripheral and central processes (Cerella, 1985). The methods proposed here are designed to test just this hypothesis and, by extension, the adequacy of a latent multiplicative slowing function in tasks in which the intercept of the molar slowing function may not be zero.

\section{Architecture of Cognition}

In this section, it will be assumed that the latent slowing function is a common, multiplicative one. Given that such is the case, one would like to know when the slowing function governing the molar response times is general, and, if so, what relation the form of the molar slowing function bears to the form of the latent slowing function. Salthouse (1978) has noted that if every process were slowed by a multiplicative factor $\beta$, then, for serial or parallel architectures, one would obtain Equation 1 with $\alpha=0$. In this case, both the latent and molar models are common, multiplicative ones. Salthouse does not go on to indicate the relation between the latent and molar slowing functions when the architecture becomes more complex, the resource demands exceed the available supply, or the durations of the processes are not independent. The effect of each of these changes on the relation between the latent and molar slowing functions will now be discussed, beginning here with an analysis of the role of the particular architecture of a network.

\section{Identical Latent and Molar Slowing Functions}

Interestingly, the form of the molar slowing function can be inferred from the form of the latent slowing functions even when the architecture is very complex. Specifically, as long as the tasks are ones with only endogenous processes arranged in a PERT network, the structures of the networks governing the behavior of older and younger adults are identical within each of the observed tasks (the network structure can vary across tasks), and as long as the latent slowing functions are common, multiplicative ones, it can be shown that the response times of older adults are one and the same multiplicative function of the response times of younger adults, regardless of the (PERT) architecture used to represent behavior in each of the 
various tasks. More formally, let $G_{\mathrm{o}}(V, E)$ be the PERT network used to represent older adults' performance, and let $G_{\mathrm{y}}(V, E)$ be the PERT network used to represent younger adults' performance within a particular task. Let $X_{j}$ represent the time that younger adults take to execute process $x_{j}$ (recall that previously $X_{j}$ was used to represent the time that the faster cohort takes to execute process $x_{j}$; this shift in the interpretation of the variable applies throughout the remainder of the article). Let $B$ represent a random slowing factor that modifies proportionately and identically the duration of all latent processes on any given trial (the randomness reflects, perhaps, varying levels of attention among older adults, something that fluctuates much more with age; Summala \& Mikkola, 1994). And let $\Psi_{j}$ represent the time that older adults take to execute process $x_{j}$ (note the same shift in the interpretation of this variable as was noted immediately above). Then,

THEOREM 1. For each task, suppose that $G_{0}(V, E)$ and $G_{\mathrm{y}}(V, E)$ are identical PERT networks, that $x_{1}, \ldots, x_{n}$ are all endogenous, that $\Psi_{j}=B X_{j}$ for $j=1, \ldots, n$, and that $B$, $X_{1}, \ldots, X_{n}$ are mutually independent. Then $E[O]=\beta E[Y]$ regardless of the task, where $E[B]=\beta$. (This theorem and the remaining theorems and corollaries are summarized in Table 3.)

The proof appears in Appendix A. A simple example can illustrate the utility of Theorem 1 . Specifically, suppose a network with two parallel processes (Figure 5) were to govern the behavior of older and younger adults in one task, and suppose an entirely different network - say, an incomplete Wheatstone bridge (Figure 4) -were to govern the behavior of older and younger adults in a second task. And suppose that the duration of each process in the older adults' network can be written as a multiple of the duration of the corresponding process in the younger adults' network, where this multiple remains constant across processes (i.e., the latent slowing function is a common, multiplicative one). Then as long as all the processes are endogenous, one and the same function will relate the molar response times of older to younger adults across the two tasks (i.e., both the molar and the latent slowing functions will be the same multiplicative constant). More generally, no matter how complex the architecture, if the latent slowing function is a common, multiplicative one, the

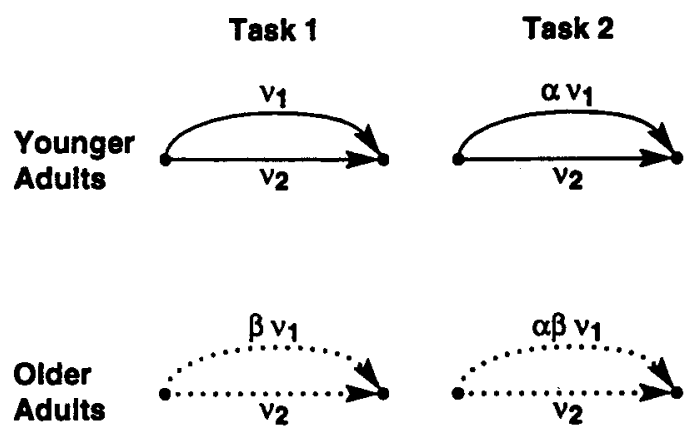

Figure 5. Parallel networks for older and younger adults in two tasks. The duration of a process $x_{j}$ labeled as $v_{j}$ is assumed to be exponentially distributed with the labeled rate parameter, and so on for the other processes.

molar slowing function will be equal to one and the same multiple as long as all processes are endogenous.

\section{Different Latent and Molar Slowing Functions}

Consider what happens if we relax the assumptions in Theorem 1. Above, for example, tasks were considered that contained only endogenous processes. Now, suppose that in order to represent behavior in a particular task, it is necessary to consider exogenous as well as endogenous processes. Then, as we have shown in the introduction, no longer will it necessarily be the case that a simple multiplicative function will relate the response times of older to younger adults across tasks even though the correct model of slowing (i.e., the latent model) is a common, multiplicative one when the process durations are constant. A simple example can indicate what is happening when the process durations vary from trial to trial. Specifically, consider again the two-process parallel network in Figure 5. However, assume now that process $x_{1}$ is endogenous, whereas process $x_{2}$ is exogenous. Participants are run in two tasks, Task 1 and Task 2.

To begin, consider just Task 1 . Suppose that the duration $X_{1}$ of process $x_{1}$ is exponential with rate parameter $v_{1}$-that is, $f\left(x_{1}\right)=v_{1} e^{-v_{1} t}$-and suppose that the duration $X_{2}$ of process $x_{2}$ is a constant equal to $v_{2}$. Since it is assumed that the latent multiplicative model of common slowing is the correct one ( $\left.\Psi_{j}=B X_{j}\right)$, the duration of pro-

Table 3

Summary of Theorems and Corollaries

\begin{tabular}{ll}
\hline Theorem 1: & no resource constraints and common multiplicative slowing function (durations) \\
Theorem 2: & $\begin{array}{l}\rightarrow \text { molar multiplicative model of general slowing } \\
\text { resource constraints and common multiplicative slowing function (rates) }\end{array}$ \\
Theorem 3: $\quad \begin{array}{l}\text { linear independence and molar multiplicative model of general slowing } \\
\text { Theorem 4: }\end{array}$ & $\begin{array}{l}\rightarrow \text { latent multiplicative model of common (but not process-specific) slowing } \\
\text { iterative tasks and molar multiplicative model of general slowing }\end{array}$ \\
Theorem 5: & $\begin{array}{l}\rightarrow \text { latent multiplicative model of common (or process-specific) slowing } \\
\rightarrow \text { molar power model of general slowing }\end{array}$ \\
Corollary 1: & $\begin{array}{l}\text { no resource constraints, dependent process durations, and common multiplicative } \\
\text { slowing function (durations) }\end{array}$ \\
& $\rightarrow$ molar multiplicative model of general slowing
\end{tabular}


cess $x_{1}$ for the older adults is exponential with rate parameter $v_{1} / \beta, \beta>1$; the duration of process $x_{2}$ does not change (e.g., the stimulus onset asynchrony, or SOA, does not change as a function of age). Then, the expected time $E\left[Y_{1}\right]$ for the younger adults to respond in Task 1 can be shown to equal

$$
E\left[Y_{1}\right]=v_{2}\left(1-e^{-v_{1} v_{2}}\right)+\left(v_{2}+\frac{1}{v_{1}}\right)\left(e^{-v_{1} v_{2}}\right) .
$$

And the expected time $E\left[O_{1}\right]$ for the older adults to respond in Task 1 can be shown to equal

$$
E\left[O_{1}\right]=v_{2}\left(1-e^{-\frac{v_{1} v_{2}}{\beta}}\right)+\left(v_{2}+\frac{\beta}{v_{1}}\right)\left(e^{-\frac{v_{1} v_{2}}{\beta}}\right) .
$$

Next, consider Task 2. Assume that in Task 2 the duration of process $x_{1}$ is delayed so that for the younger adults it is exponentially distributed with rate parameter $v_{1} / \alpha$, $\alpha>1$. The duration of process $x_{1}$ for the older adults will now be exponentially distributed with rate parameter $v_{1} /(\alpha \beta)$; again, since process $x_{2}$ is exogenous, the duration of this process does not change. Similar to what is shown above, in Task 2 the expected response time $E\left[Y_{2}\right]$ for the younger adults and the expected response time for the older adults $E\left[\mathrm{O}_{2}\right]$ are computed as follows:

$$
\begin{aligned}
& E\left[Y_{2}\right]=v_{2}\left(1-e^{-\frac{v_{1} v_{2}}{\alpha}}\right)+\left(v_{2}+\frac{\alpha}{v_{1}}\right)\left(e^{\left.-\frac{v_{1} v_{2}}{\alpha}\right),}\right. \\
& E\left[O_{2}\right]=v_{2}\left(1-e^{-\frac{v_{1} v_{2}}{\alpha \beta}}\right)+\left(v_{2}+\frac{\alpha \beta}{v_{1}}\right)\left(e^{-\frac{v_{1} v_{2}}{\alpha \beta}}\right) .
\end{aligned}
$$

It follows simply that when one or more processes are exogenous, in general it will not be the case that the response times of older adults can be expressed as the same multiple of the response times of younger adults across tasks (i.e., $E\left[O_{1}\right] / E\left[Y_{1}\right] \neq E\left[O_{2}\right] / E\left[Y_{2}\right]$ ), even though the latent multiplicative model of common slowing governs behavior. For example, in Figure 5, if $v_{1}=v_{2}=1$ and $\alpha=$ $\beta=2$, then $E\left[O_{1}\right] / E\left[Y_{1}\right]=1.62$ and $E\left[O_{2}\right] / E\left[Y_{2}\right]=1.86$.

The number of published studies in which it appears that the model of general slowing may have been falsely rejected is presumably reasonably small, in part because only recently has there been a serious attempt to investigate in detail alternatives to the model of general slowing. However, caution is still required. For example, the authors of one recent study undertook a meta-analysis of the results from 15 semantic priming experiments (Laver \& Burke, 1993). They concluded that the slowing was not general. This may indeed be the case at the molar level. But, semantic priming experiments such as Laver and Burke analyzed typically include an exogenous process, the interval of time between the presentation of the prime and the target. As has been argued above, the molar model of slowing need not be a general, multiplicative one even though the latent model of slowing is a common, multiplicative one if the network governing performance includes one or more exogenous processes. Thus, the results from the studies of semantic priming could well be consistent with a latent model of common slowing in which all processes are slowed identically, even though they are not consistent with a molar model of general slowing.

\section{Resource Constraints}

Just as it would appear to be unrealistic to assume that performance in all (or even most) tasks can be represented in simple serial or parallel networks, so too it would appear to be unrealistic to assume that the rate at which a given process is being executed is independent of the other processes executing at the same time. Rather, if there exists a fixed capacity, then every time a process is completed the capacity may be redistributed, and, as a consequence, the work rates may be set anew. For example, in a visual search task, the available capacity may be divided initially among all of the stimuli in the display. If there were four stimuli and four total resources, each stimulus could be allocated initially one unit of resources, and the work rate could be computed accordingly. However, as soon as it is determined that one of the stimuli is not the target, the available capacity can be redistributed among the remaining stimuli (Townsend \& Ashby, 1983, pp. 138-139). In the present visual search example, this would mean that the four resources should now get redistributed among three stimuli, presumably increasing thereby the rate at which the processing of each stimulus can be completed. This resetting reflects a not unreasonable reallocation of resources that might occur as one process goes off line (and perhaps other processes come on line).

This same interpretation of the relation between resources, work rates, and processing time can be used to explain the differences in younger and older adults' performance. Specifically, if older adults have fewer resources than younger adults do, they will work on a given process more slowly and thus take longer to complete the process. An example can indicate more concretely what might happen. Suppose it requires 10 units of work by both older and younger adults to complete a task. And suppose that younger adults have 10 total resources, each of which can be used to complete one unit of work per second, whereas older adults have only 5 total resources. Then younger adults can complete the task in $1 \mathrm{sec}$, whereas older adults require a full $2 \mathrm{sec}$.

The question, then, is just how the existence of resource constraints will affect the relation between the latent and molar models of slowing. There are many different ways one might model resource constraints that capture the dependence of work rates (and therefore processing times) on the resource constraints. Goldstein and Fisher (1992) have recently suggested a method that is fairly general and can be used to better understand the relation between the latent and molar models. In order to explain their method, it will be necessary to describe very briefly what they have called an order-of-processing (OP) diagram (Fisher \& Goldstein, 1983). An example should speed the explanation. In particular, suppose that two processes, $x_{1}$ 
and $x_{2}$, are executing in parallel (Figure 6a). At any moment in the unfolding of the network over time, it is possible to partition these two processes into one of three sets: the preactive, current, and completed sets. The preactive set contains the processes that have not yet started executing; the current set contains the processes that are now executing; and the completed set contains the processes that have finished executing. Together, each partition forms a state in the OP diagram. Arcs connect states that directly follow one another in time.

For example, the OP diagram representation of the two parallel processes is represented in Figure 6b. Processes in the current set of a state are placed in the upper half of the state. Processes in the completed set are placed in the lower half. Processes in the preactive set can be obtained by subtraction and so are not listed. At the outset (state $s_{1}$ ), both processes are current, and so both are listed above the horizontal line in the first state $\left(s_{1}\right)$. No processes are preactive (both processes $x_{1}$ and $x_{2}$ begin executing immediately). No processes are completed. After some time, either process $x_{1}$ or process $x_{2}$ will finish. If process $x_{1}$ finishes first, a new partition (state) comes into existence. Specifically, now process $x_{1}$ is in the completed set (below the horizontal line in state $s_{2}$ ), and process $x_{2}$ remains in the current set (above the horizontal line in state $s_{2}$ ). The remaining states in the OP diagram are constructed similarly. Note that the process that finishes when a transition is made between two states is listed beside (beneath or above) the arc connecting the two states.

The gist of the Goldstein and Fisher (1992) implementation of resource constraints can now be made clear. Specifically, they assume that the work $W_{j}$ required in order to complete each process $x_{j}$ with duration $X_{j}$ is a random

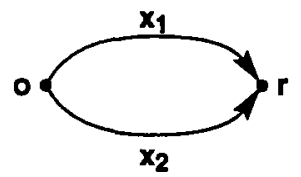

(a)

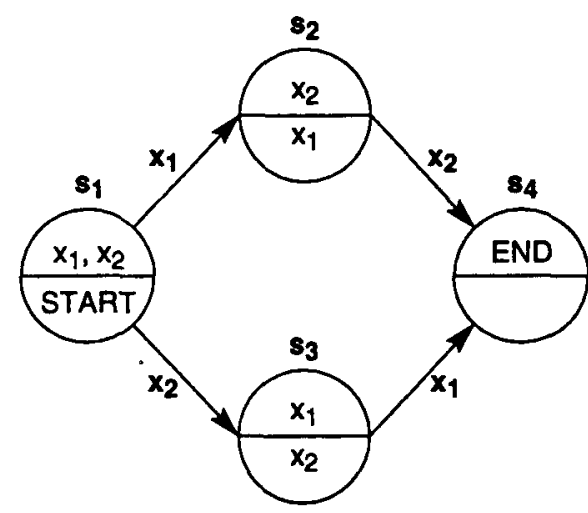

(b)

Figure 6. A two-process parallel PERT network (a) and corresponding OP diagram (b). variable, where $W_{j}=\int_{0}^{X_{j}} v_{j}(t) d t$ and where the rate $v_{j}(t)$ changes only when a transition is made from one state to the other. Let the complete set of work requirements be represented by the random vector $\mathbf{W}=\left(W_{1}, \ldots, W_{n}\right)^{\prime}$ (the prime indicates that the list of variables that occurs between the parentheses is a column vector). The work done on the $j$ th process in the $k$ th state of the $h$ th path in the OP diagram is equal to the product of the rate $v(j, k, h)$ at which work proceeds on the process times the duration $T_{h_{k}}$ of the state, where the rate parameter $v(j, k, h)$ is a function of the process $x_{j}$ and the first $k$ states along path $h$. [Note that here $h$ is used to index paths in the OP diagram, whereas above it was used to index paths in a PERT network; here $k$ is used to index the states along a path in the OP diagram, whereas above it was used to index processes along a path in the PERT network; and here $n(h)$ refers to the number of states along a path $h$ in the OP diagram, whereas above it was used to index the number of processes on a path in the PERT network. The intended use will be clear from the context.] Thus, for example, if an individual can do work at the rate of 5 units/sec on a particular process in a particular state, and if the state is $5 \mathrm{sec}$ in duration, the work done is 25 units. More generally, the $W_{j}$ work done on the $j$ th process along all states on the $h$ th path can be written as follows:

$$
W_{j}=\sum_{k=1}^{n(h)} v(j, k, h) T_{h_{k}}
$$

where $v(j, k, h)=0$ if process $x_{j}$ is not in the current set of state $s_{h_{k}}$ (see Townsend \& Ashby for a related formulation; 1983, pp. 77-79).

This formulation leads directly to the desired restrictions on resources. For example, consider the rate at which work is completed on the processes current in state $s_{1}$ of Figure $6 \mathrm{~b}$. Since two processes $\left(x_{1}\right.$ and $\left.x_{2}\right)$ are competing for resources in this state, the associated work rates, $v(1,1, h)$ and $v(2,1, h)$, might well be much slower than the work rates when either process $x_{2}$ (in state $s_{2}$ ) or process $x_{1}$ (in state $s_{3}$ ) is executed by itself. This dependence of the work rates on the particular set of processes executing in a state is easily handled in the resource model outlined above, since the work rates $v(j, k, h)$ are a function of the state in which processing is ongoing.

\section{Related (Reciprocal) Latent and Molar Slowing Functions}

Clearly, the introduction of resource constraints can greatly complicate the mathematics of the underlying latent model. However, in many cases, this additional complication will not change the relation between the latent and molar models. Specifically, assume that the work required to complete the processes in a task has the same distribution for younger adults that it has for older adults; that is, assume that $P\left(\mathbf{W}_{\mathrm{o}} \leq \mathbf{w}\right)=P\left(\mathbf{W}_{\mathrm{y}} \leq \mathbf{w}\right)$, where $\mathbf{W}_{\mathrm{o}}$ is the random vector of older adult work requirements, $\mathbf{W}_{\mathrm{y}}$ is the random vector of younger adult work requirements, and $\mathbf{w}$ is a vector of non-negative constants. And, assume that the rate at which older adults work on a particular 
process $x_{j}$ in a particular state $k$ having been reached along a particular path $h$ is slowed by a proportion $\beta$ that is independent of the process, task, and path; that is, assume that $v_{\mathrm{o}}(j, k, h)=\beta v_{\mathrm{y}}(j, k, h), \beta<1$ for all processes, states, and paths. The resource allocation policies of the older and younger adults are identical in this case. That is, the proportion of the total resources allocated to each process in a given state (as indexed by the ratio of the rate of a given process to the sum of the rates of all processes executing in the specified state) is the same for both older and younger adults.

Note that the type of slowing function that we are proposing for resource-constrained networks can still (conveniently) be referred to as a common, multiplicative latent slowing function. However, in this context (where resources are constrained), to say that the latent slowing function is a common, multiplicative one is to imply that the work rates of the older adults are decreased by some proportion $\beta$ less than one (across all processes, states, and paths), whereas in the previous context (where resources were not constrained), to say that the latent slowing function is a common, multiplicative one is to imply that the process durations of older adults are increased by some proportion $\beta$ greater than one (across all processes).

If the preceding assumptions about the relation between the work rates and distribution of work requirements of younger and older adults hold, if all processes used to represent a task are endogenous ones arranged in a PERT network, and if the networks for the older and younger adults are the same within tasks, it can be shown that the older adults' response times can be written as a multiple of the younger adults' response times, regardless of how the resources are distributed among the processes in each state. More formally,

THEOREM 2. For each task, suppose that $G_{0}(V, E)$ and $G_{\mathrm{y}}(V, E)$ are identical PERT networks, that $x_{1}, \ldots, x_{n}$ are all endogenous, that $P\left(\mathbf{W}_{\mathrm{o}} \leq \mathbf{w}\right)=P\left(\mathbf{W}_{\mathrm{y}} \leq \mathbf{w}\right)$, and that $v_{\mathrm{o}}(j, k, h)=\beta v_{\mathrm{y}}(j, k, h), \beta<1$, for all processes $j$, states $k$, and paths $h$. Then $E[O]=\beta^{-1} E[Y]$, regardless of the task.

The proof appears in Appendix B. An extension of an example at the end of the previous section can illustrate the utility of this theorem. In particular, assume that the network governing the performance of older and younger adults is parallel in one task (Figure 5) and an incomplete Wheatstone bridge in another (Figure 4). Assume that older adults must do the same work as must younger adults to complete each process. Assume that the work rates change only when a process finishes. And assume that the older adults complete the work on any given process in a particular state at some common reduced rate because they have access to fewer total resources. Then, as long as all processes are endogenous, across the two tasks one factor will relate the overall response times of older to younger adults, and the reciprocal of this factor will relate the work rates of older to younger adults. More generally, no matter how complex the architecture, if the assumptions of Theorem 2 are satisfied, and, in particular, if the latent slowing factor (in this case, the factor that reduces the rate at which work proceeds on a particular process in a particular state) is a common, multiplicative one, the molar slowing factor will be equal to the reciprocal of the latent slowing factor as long as all processes are endogenous.

\section{Unrelated Latent and Molar Slowing Functions}

Consider what happens if we relax the assumptions in Theorem 2. In particular, consider what happens if the work requirements of the older and younger adults are not identical in at least one task. For example, consider two tasks, each with three processes arranged in series. Assume that the work requirements of the older and younger adults are identical in the first task. Then, by Theorem 2, $E\left[O_{1}\right]=\beta^{-1} E\left[Y_{1}\right]$. Now, assume for the sake of simplicity that the older adults require $\alpha$ times as much work in order to complete the three processes in the second task. Then, $E\left[O_{2}\right]=\alpha \beta^{-1} E\left[Y_{2}\right]$. Clearly, the proportionate slowing of the overall response times of the younger adults in the first task (by a factor of $\beta^{-1}$ ) does not equal the proportionate slowing in the second task $\left(\alpha \beta^{-1}\right)$.

Finally, consider what can happen if the work rates change at times other than the completion of a process (i.e., other than the transition between states in the OP diagram). Intuitively, if all work rates are lengthened by the same amount throughout the duration of a state, the process that finishes first in a particular state in the OP diagram for a given assignment of work rates is the same in the cohort without a reduction in work rates as it is in the cohort with a reduction in work rates. Thus, the probability that a particular path is taken remains the same for the two cohorts, and the state durations are lengthened by the reciprocal of the work rate in the slower cohort. However, if the work rates change sometime in the middle of a state, the state durations of one cohort no longer bear the neat relation to the state durations of the related cohort. For example, consider a task with just one process and a work requirement of 10 units. Since there is only one process, there is only one state in which a process is current in the OP diagram. Assume that, during the first second, 5 units can be completed $\left[v_{\mathrm{y}}(t)=5,0 \leq t \leq 1\right]$. And assume that, during the next $5 \mathrm{sec}$, the remaining 5 units can be completed $\left[v_{\mathrm{y}}(t)=1,1<t\right]$. Thus, the state (and task) duration is $6 \mathrm{sec}$. Now, suppose that for the older cohort we decrease the work rates by $50 \%\left[v_{0}(t)=2.5\right.$, $\left.0 \leq t \leq 1 ; v_{0}(t)=.5,1<t\right]$. Then, during the first second, 2.5 units can be completed. During the remaining $15 \mathrm{sec}$, the final 7.5 units can be completed. Thus, the state (task) has a total duration of $17.5 \mathrm{sec}$, far more than double the state (task) duration when the work rates are not reduced.

\section{Dependencies}

As a final possibility, consider the relation between molar and latent models when dependencies exist among the process durations. Above, it was assumed that the durations of the processes were mutually independent. Clearly such will not always be the case. For example, if 
a subject's attention wanders during a trial, one would expect the durations of several of the latent processes to be lengthened during that trial. Similarly, if a subject were generally alert on one day, but not on a second day, one would expect the durations of a subset of the processes to be relatively short on the first day and relatively long on the second. Note that unlike in the previous section, we are assuming here (and until stated otherwise) that there are no resource constraints. Thus, the latent slowing function applies to the process durations of the younger adults (and not the work rates).

\section{Identical Latent and Molar Slowing Functions}

First, we want to allow for the possibility that the duration of one or more processes depends on the durations of some other processes. Let $\mathbf{X}$ be the random vector $\left(X_{1}\right.$, $\left.\ldots, X_{n}\right)^{\prime}$. And let $\Sigma$ be the covariance matrix, where some of the off-diagonal entries in the covariance matrix are not equal to zero (implying that the durations of some processes are dependent). Second, we want to allow for the possibility that separate covariance matrices might govern the behavior of older and younger adults. Let $\Sigma_{0}$ be the covariance matrix for older adults and let $\Sigma_{y}$ be the covariance matrix for younger adults. Then, regardless of the nature or complexity of the dependencies that might exist among the process durations, as long as the covariance matrices of the older and younger adults are identical, the molar and latent slowing functions are identical. More formally,

COROllary 1. For each task, suppose that $G_{0}(V, E)$ and $G_{\mathrm{y}}(V, E)$ are identical PERT networks, that $x_{1}, \ldots, x_{n}$ are all endogenous, that $\Psi_{j}=B X_{j}$, that $\Sigma_{\mathrm{o}}$ and $\Sigma_{\mathrm{y}}$ are identical, and that $B$ is mutually independent of $X_{j}$ for $j=1, \ldots, n$. Then $E[O]=\beta E[Y]$, regardless of the task where $E[B]=\beta$.

The proof follows directly from Theorem 1 and is left as an exercise for the reader. Note that this corollary generalizes Theorem 1. In particular, in tasks in which the duration of one process depends on the duration of some other process, if the latent slowing function is a common, multiplicative one, the molar slowing function will be a general, multiplicative one. However, we need to be careful to ensure that the pattern of dependencies among the process durations are identical for older and younger adults.

\section{Different Latent and Molar Slowing Functions}

Consider what happens if we relax the assumptions in Corollary 1. Specifically, consider what happens if a different set of dependencies govern the durations of the constituent processes. A simple example can indicate why it is important to make sure whenever possible that the dependencies are similar. Suppose that two processes are in parallel and that each process takes $1 \mathrm{sec}$ with probability $1 / 2$ and $2 \mathrm{sec}$ with probability $1 / 2$. Assume that the durations of the processes are independent for the younger adults. Then, the expected time to complete both processes (i.e., the last finishing process) is $1.75 \mathrm{sec}$. Now, assume that the durations of the two processes are dependent among the older adults. Specifically, assume that the two processes each take $1.1 \mathrm{sec}$ with probability $1 / 2$ and $2.2 \mathrm{sec}$ with probability $1 / 2$. Thus, the average duration of each process has increased from $1.5 \mathrm{sec}$ in the younger adults to $1.65 \mathrm{sec}$ in the older adults. However, the expected time to complete both processes for the older adults $(1.65 \mathrm{sec})$ is less than the expected time to complete both processes for the younger adults $(1.75 \mathrm{sec})$. Thus, when dependencies exist among the process durations, the response times of the older adults could well be less than the response times of the younger adults in any given task, even though the networks governing the performance of the older and younger adults' behavior are identical and the average durations of the individual processes in the older adults' network are longer than the average durations of the individual processes in the younger adults' network.

\section{LOGICAL ANALYSES: FALSE POSITIVE DECISIONS}

In the preceding section, we assumed that we knew the identity of the latent slowing function and then derived the identity of the molar slowing function. From this, we could determine when we might expect falsely to reject the latent model of common slowing if we based our rejection solely on the knowledge that the molar model of general slowing was not the correct one. In this section, we want to assume that we know the identity of the molar slowing function and then attempt to derive the identity of the latent slowing function(s). From this, we will be able to determine when we might expect falsely to accept the latent model of common slowing if we based our acceptance solely on the knowledge that the molar model of general slowing was the correct one.

\section{Unique Common Latent Slowing Function}

Just as we needed to assume something about the structure of the underlying network, the nature of the resource constraints, and the dependencies among the process durations before we could make any progress relating the latent to the molar slowing function, so too we will need to assume something about the structure of the underlying network, the nature of the resource constraints, and the dependencies among the process durations before we can make any progress relating the molar to the latent slowing functions. To begin, we want to assume that for each of $m$ tasks the network is serial, there are no resource constraints, and the process durations are independent. And we want to assume that the number of unique latent processes is less than or equal to the number of tasks. Specifically, let $\mathbf{N}_{i}$ represent the set of latent processes in task $i$. Let $n$ in this context represent the number of unique processes in the union of sets $\mathbf{N}_{1}, \ldots, \mathbf{N}_{m}$. For example, suppose that there are 5 tasks, where Task 1 was composed of latent processes $x_{1}, x_{3}$, and $x_{4}$, Task 2 was composed of latent processes $x_{2}$ and $x_{4}$, Task 3 was composed of processes $x_{1}$ and $x_{2}$, Task 4 was composed of 
processes $x_{2}, x_{3}$, and $x_{4}$, and Task 5 was composed of processes $x_{1}$ and $x_{4}$. Then $n=4$ and $m=5$.

If these very general conditions are satisfied, and if the molar slowing function is a general, multiplicative one, we can specify under what circumstances the latent function will necessarily be a common, multiplicative one. Let $\mu_{i j}$ represent the expected duration of process $x_{j}$ in task $i$, where $\mu_{i j}=0$ if process $x_{j}$ is not present in task $i$. Let $\Lambda$ represent the $m \times n$ matrix $\left[\mu_{i j}\right]$ of process durations. Then, if the columns of the process duration matrix $\Lambda$ are linearly independent, the latent slowing function must be a common, multiplicative one:

THEOREM 3. Suppose that $E\left[O_{i}\right]=\beta E\left[Y_{i}\right]$ for all $m$ tasks, that $\Psi_{j}=\beta_{j} X_{j}$ for all $n$ unique processes $x_{1}, \ldots, x_{n}$, and that the rank of $\Lambda$ is equal to $n, m \geq n$. Further, for each task, suppose that $G_{\mathrm{o}}(V, E)$ and $G_{\mathrm{y}}(V, E)$ are identical serial networks and that the $n$ processes are all endogenous. Then only the latent model of common slowing is consistent with the molar model of general slowing; that is, the molar model of general slowing implies $\beta_{j}=\beta$ for all $j$.

The proof is in Appendix C. Computationally, it is easy to determine whether the columns of the process duration matrix are linearly independent and therefore whether the latent model of common slowing is the only one consistent with a molar model of common slowing. Practically, the difficulties in applying this theorem may come in estimating the expected durations of each of the $n$ unique latent processes. Fortunately, we can easily recognize many of the experiments in which there is a good chance that the columns of the process duration matrix are linearly dependent and therefore both a latent model of common slowing and a latent model of process-specific slowing are consistent with a molar model of general slowing. In this case, one cannot logically infer that the only latent slowing function consistent with the molar slowing function is a common one. Rather latent slowing functions could be consistent with the molar slowing function which are process specific. This problem is similar to both the nonidentifiability of parallel and serial models in reaction time studies (Townsend, 1972) and the nonidentifiability of certain alternative state models in learning and memory studies (Greeno \& Steiner, 1964). Often, models that reflect very different ways of processing information cannot be differentiated on the basis of one or more summary statistics.

\section{Equivalent Common and Process-Specific Slowing Functions}

The types of tasks in which one might find that a latent model of process-specific slowing could mimic a latent model of common slowing were first described by Fisk and Fisher (1994) and later elaborated upon by Hale et al. (1995). One such example task was described in the introduction. We now want to generalize the example. Briefly, suppose that each of the $m$ tasks requires the execution of all $n$ unique processes. And suppose that the durations of the latent processes in one task are proportional to the durations of the latent processes in any other task; that is, $\mu_{i j} / \mu_{i^{\prime} j}=\mu_{i j^{\prime}} / \mu_{i^{\prime} j^{\prime}}, j, j^{\prime}=1, \ldots, n, i, i^{\prime}=1, \ldots, m$, assuming that processes $j$ and $j^{\prime}$ occur in both tasks $i$ and $i^{\prime}$. This might occur if in each task the entire set of latent processes needed to be repeated several times in order to complete the task, the exact number of repetitions being the characteristic that differentiated one task from another. Hale et al. refer to such tasks as iterative tasks. Then, for an iterative set of tasks, the latent model of processspecific slowing can be made to predict the same relation between the overall response times of older and younger adults as does the latent model of common slowing. Specifically,

TheOREM 4. Suppose that $E\left[O_{i}\right]=\beta E\left[Y_{i}\right]$ for all $m$ tasks, that $\Psi_{j}=\beta_{j} X_{j}$ for all $n$ unique latent processes $x_{1}$, $\ldots, x_{n}$, and that the tasks are iterative. Further, for each task, suppose that $G_{\mathrm{o}}(V, E)$ and $G_{\mathrm{y}}(V, E)$ are identical serial networks each with $n$ latent processes, and that the $n$ processes are all endogenous. Then both a latent model of process-specific and a latent model of common slowing are consistent with the molar model of general slowing.

The proof of this theorem is given in Appendix D. An example can give some substance to Theorem 4 . In particular, assume that there are three tasks. Additionally, assume that the time $E\left[Y_{i}\right]$ on the average that it takes younger adults to respond in task $i$ is equal to the sum of the average duration $E\left[Y_{i c}\right]$ of the central processes plus the average duration $E\left[Y_{i p}\right]$ of the peripheral processes; that is, $E\left[Y_{i}\right]=E\left[Y_{i c}\right]+E\left[Y_{i p}\right]$. Set $E\left[Y_{i c}\right]=\gamma_{i} 900$ and $E\left[Y_{i p}\right]=\gamma_{i} 100$, where $\gamma_{i}=i, i=1,2,3$. Finally, set $\beta=2$, $\beta_{c}=1$, and $\beta_{p}=11$, where $\beta$ is the common slowing factor in the molar model of general slowing $\left(E\left[O_{i}\right]=\beta E\left[Y_{i}\right]\right)$ and where $\beta_{c}$ and $\beta_{p}$ are, respectively, the central and peripheral process slowing factors in the latent model of process-specific slowing $\left(E\left[O_{i}\right]=\beta_{c} E\left[Y_{i c}\right]+\beta_{p} E\left[Y_{i p}\right]\right)$. Then, Task 1 takes $1,000 \mathrm{msec}$ for the younger adults $(900+100)$. If the molar model of general slowing is the correct one, it should take $2(900+100)=2,000 \mathrm{msec}$ for the older adults. And if the latent model of processspecific slowing is the correct one, it should take $1(900)+$ $11(100)=2,000 \mathrm{msec}$ also for the older adults (Table 4). Similarly, it is clear that in Tasks 2 and 3 the predictions of the models of general and process-specific slowing are identical.

Other authors have shown that a latent multiplicative model of process-specific slowing can be rewritten as a molar linear model of general slowing. For example, as noted earlier, Cerella (1985) shows that the negative intercept sometimes seen when molar linear models of general slowing are fit to older and younger adults' response times can arise from a particular form of a latent multiplicative model of process-specific slowing. However, the emphasis in his (and others') work has not been on the potential for falsely accepting a model of general slowing when it should be rejected. 
Table 4

Predictions of General and Process-Specific Models

\begin{tabular}{|c|c|c|c|}
\hline \multirow[b]{3}{*}{ Processes } & \multicolumn{3}{|c|}{ RTs } \\
\hline & \multirow[b]{2}{*}{ Young } & \multicolumn{2}{|c|}{ Old } \\
\hline & & General & Specific \\
\hline \multicolumn{4}{|c|}{ Task 1} \\
\hline Central & 900 & 1,800 & 900 \\
\hline Peripheral & 100 & 200 & 1,100 \\
\hline Total & 1,000 & 2,000 & 2,000 \\
\hline \multicolumn{4}{|c|}{ Task 2} \\
\hline Central & 1,800 & 3,600 & 1,800 \\
\hline Peripheral & 200 & 400 & 2,200 \\
\hline Total & 2,000 & 4,000 & 4,000 \\
\hline \multicolumn{4}{|c|}{ Task 3} \\
\hline Central & 2,700 & 5,400 & 2,700 \\
\hline Peripheral & 300 & 600 & 3,300 \\
\hline Total & 3,000 & 6,000 & 6,000 \\
\hline
\end{tabular}

\section{STATISTICAL ANALYSES: FALSE POSITIVE DECISIONS}

Above, it was assumed that we knew whether the molar model of slowing was or was not a general one. Of course, we generally do not know this and must, instead, test this assumption. We want now to talk briefly about how one might construct an appropriate test. The molar linear models of general slowing are typically analyzed by plotting the response times of older adults against those of younger adults and determining the percentage of variance explained by the best fitting line. However, as noted in the introduction, this type of analysis can easily lead to the decision falsely to accept the molar model of general slowing on the basis of the very good fit of such a model. As one possible alternative analysis, it seems straightforward enough to regress the response times of older adults on the response times of younger adults in each task and then compare the regression parameters across tasks (Myerson, Wagstaff, \& Hale, 1994). However, here the independent variable (the response time of younger adults) is random and will almost certainly contain some measurement errors. This may create problems for the proposed analysis (Neter \& Wasserman, 1974, pp. 168-169; although see Myerson et al., 1994). Thus, an alternative test may be required. One very general test is described immediately below. This test, unlike other more simple tests, can be used to estimate the actual slowing functions of various of the constituent processes that might otherwise not be available for inspection.

It is easiest to explain the alternative test in the context of a particular example analysis. We want to use the results from a memory search task reported by Salthouse and Somberg (1982; adapted from Sternberg, 1969). Briefly, in the memory search task, participants were given a list of either one or four digits to memorize at the start of each trial. The digits were then erased from the screen, and after a short interval of time a new target digit appeared. Subjects were asked to indicate whether the tar- get digit was or was not in the memory set and, in the condition requiring a complex response, to identify the target digit. Finally, the target digit was displayed in either an intact or a degraded condition.

To implement the test, we first need to construct a latent model of processing for the younger and older adults. Such a model has already been developed (Sternberg, 1969). Specifically, it is assumed that the participants initially encode the target stimulus, then compare this stimulus one at a time with all memory set stimuli, and finally respond. (Note that the response times are collapsed across target present and target absent trials, so that there is no need for a stage representing the target present or target absent conditions.) Second, one must test this latent informationprocessing model in each cohort. Again, the model has been tested across a wide range of experiments and cohorts (e.g., Eriksen, Hamlin, \& Daye, 1973; Sternberg, 1969). Finally, one needs to determine whether a molar model of general slowing or a latent model of process-specific slowing better captures the affects of aging on performance. In order to make this last determination, one needs to predict performance in each of the relevant conditions. In this context, a molar model of general slowing and a latent model of common slowing make identical predictions (Theorem 1). Thus, below we will be comparing the fit of the latent models of common and process-specific slowing.

The predictions for the two latent models can now be derived quite simply. For younger adults, let $E_{e}, C_{c}$, and $R_{r}$ be the durations of, respectively, the encoding stage at level $e$ ( $e=0$ if intact, $e=1$ if degraded), the comparison stage at level $c$ ( $c=0$ if one stimulus is in the memory set, $c=1$ if four stimuli are in the memory set), and the response stage at level $r(r=0$ if the response is simple, $r=1$ if the response is complex). Then, if the latent multiplicative model of slowing is a common one, the average time that it takes younger and older adults to respond can be written as follows:

$$
\begin{aligned}
& E\left[Y_{e c r}\right]=E\left[E_{e}+C_{c}+R_{r}\right], \\
& E\left[Y_{e c r}\right]=E\left[\beta\left(E_{e}+C_{c}+R_{r}\right)\right] .
\end{aligned}
$$

However, if the latent multiplicative model of slowing is a process-specific one, then

$$
\begin{aligned}
& E\left[Y_{e c r}\right]=E\left[E_{e}+C_{c}+R_{r}\right], \\
& E\left[O_{e c r}\right]=E\left[\beta_{e} E_{e}+\beta_{c} C_{c}+\beta_{r} R_{r}\right] .
\end{aligned}
$$

To determine whether the latent model of processspecific slowing explains significantly more variance in the response times than the latent model of common slowing explains, we now need to estimate the relevant parameters. Specifically, if the latent model of common slowing is the correct one, we need to estimate six parameters: the slowing factor $\beta$ and the expected durations, respectively, of the intact and degraded decoding times, the simple and complex response times, and the single-stimulus comparison time (note that the time to compare four stimuli is equal to four times the time it takes to compare a single stimulus). Salthouse and Somberg (1982) do not re- 
port the means for all eight conditions for both younger and older adults. Instead, for each cohort, they report only six means-in particular, the mean times in the intact and degraded conditions, in the small (one stimulus) and large (four stimuli) memory set size conditions, and in the mean simple and complex response conditions. The six predictions of these means for each cohort of older and younger adults can easily be obtained from Equation 15 above. Let $O_{0}$ be the time on the average in the intact encoding condition; let $O_{1}$.. be the time on the average in the degraded encoding condition; and so on. Then,

$$
E\left[O_{0 . .}\right]=\frac{E\left[O_{000}\right]+E\left[O_{001}\right]+E\left[O_{010}\right]+E\left[O_{011}\right]}{4} \text {. }
$$

And so on. Parameter values were chosen which minimize the error sum of squares $S S E(\mathrm{CS})$ for the latent model of common slowing:

$$
\begin{aligned}
\operatorname{SSE}(\mathrm{CS})= & \sum_{e=0}^{1}\left(O_{e . .}-E\left[O_{e . .}\right]\right)^{2}+\sum_{c=0}^{1}\left(O_{. c .}-E\left[O_{. c .}\right]\right)^{2} \\
& +\sum_{r=0}^{1}\left(O_{. . r}-E\left[O_{. . r}\right]\right)^{2}+\sum_{e=0}^{1}\left(Y_{e . .}-E\left[Y_{e . .}\right]\right)^{2} \\
& +\sum_{c=0}^{1}\left(Y_{. c .}-E\left[Y_{. c}\right]\right)^{2}+\sum_{r=0}^{1}\left(Y_{. . r}-E\left[Y_{. . r}\right]\right)^{2}
\end{aligned}
$$

If the latent model of process-specific slowing is the correct one, we need to estimate eight parameters: both the slowing factors $\beta_{e}, \beta_{c}$, and $\beta_{r}$ for, respectively, the encoding, comparison, and response processes, and the expected durations of, respectively, the intact and degraded encoding times, the simple and complex times, and the single-stimulus comparison time. The six predictions are obtained from Equation 16. Parameter values were chosen which minimize the error sum of squares $S S E$ (PSS) for the process-specific model.

We can now determine whether the fit of the latent model of process-specific slowing is significantly better than the fit of the latent model of common slowing. Let $d f(\mathrm{CS})$ equal the degrees of freedom for the latent model of common slowing: $d f(\mathrm{CS})=12-6$. And let $d f(\mathrm{PSS})$ equal the degrees of freedom for the latent model of process-specific slowing: $d f(\mathrm{PSS})=12-8$. Then, the statistic (Neter \& Wasserman, 1974, p. 88, Equation 3.66),

$$
\frac{\left(\frac{S S E(\mathrm{CS})-S S E(\mathrm{PSS})}{d f(\mathrm{CS})-d f(\mathrm{PSS})}\right)}{\left(\frac{S S E(\mathrm{PSS})}{d f(\mathrm{PSS})}\right)},
$$

has an $F$ distribution with $d f(\mathrm{CS})-d f(\mathrm{PSS})$ degrees of freedom in the numerator and $d f$ (PSS) degrees of freedom in the denominator if the latent model of common slowing is the correct one (assuming that the errors are normally distributed). If this statistic is significant at an appropriate level, we can reject the latent model of common slowing.

With the use of a simple grid search to minimize the error sum of squares, it was found that $\operatorname{SSE}(\mathrm{CS})=7,393$ and $S S E$ (PSS) $=1,704$, yielding an $F$ of 12.16. The $p$ value is .056 . The best fitting values for the slowing coefficients in the process-specific model were $\beta_{e}=1.6$, $\beta_{c}=1.5$, and $\beta_{r}=2.2$. The value of the slowing coefficient in the latent model of common slowing was 1.8. Clearly the range of slowing parameters is reasonable. Three things are noteworthy. First, the slowing of the response process is nearly $50 \%$ greater than the slowing of the comparison process (i.e., $\beta_{c}=1.5, \beta_{r}=2.2$ ). We would argue that this is a difference of considerable relative magnitude. Second, note that this large difference is completely hidden when just the fit of the latent model of common slowing is examined. Specifically, the latent model of common slowing explains fully $98 \%$ of the variance in the response times. Thus, it is not at all unlikely that someone looking at this fit might conclude that the slowing functions were essentially identical. Finally, note that the finding of a greater slowing of the decision and response processes is consistent with recent results from cognitive psychological studies (see Bashore, 1993, for a review).

It should be emphasized that care must be taken when using the above statistic in ways other than that described. For example, Lorch and Myers (1990) showed that repeated measures designs with nonorthogonal factors complicate the test of whether there is or is not an increase in response times across changes in the level of one of the experimental factors. In particular, they note an increase in the number of false alarms in such designs when standard tests are used and the slope is allowed to vary from one subject to next. The same increase in false alarms would be noted if one were to use the test that we are proposing to evaluate the hypothesis upon which Lorch and Myers focus. However, we are interested in a different hypothesis. In particular, we want to know whether the slowing factor is a common one or instead several different process-specific slowing factors are needed. We used simulations to determine whether in the test that we are proposing for a common slowing factor the presence of variability from one subject to the next in the slowing function had any effect on the number of false alarms. It did not. Still, it is clear that more work is needed in order to determine analytically whether the use of the same subjects across different levels of a factor compromises the test that we have proposed.

\section{STATISTICAL ANALYSES: FALSE NEGATIVE DECISIONS}

Above, it was argued that even if a latent model of common slowing fits very well, it is still necessary to test a latent model of process-specific slowing. Only by doing such can one avoid falsely accepting the latent model of common slowing (and the associated molar model of general slowing). Here it will be argued that even if a molar model of general slowing fits relatively poorly (relative 
to a molar model of task-specific slowing), it is still necessary to test a latent model of common slowing. In this case, it is only by doing such that one will avoid falsely rejecting a latent model of common slowing.

A hypothetical example that addresses this point has already been presented. In particular, it was shown earlier that the multiplicative slowing constant in one task could easily differ from the multiplicative slowing constant in a second task, even though the underlying or latent model was a common one. Thus, a molar multiplicative model of task-specific slowing provides a better fit than a molar multiplicative model of general slowing. However, the slowing constants differed by a relatively small amount ( 1.62 in one task, 1.86 in a second task), and so the effect was not dramatic. Furthermore, observations were gathered in only two conditions, so that the molar linear model of general slowing would have explained all of the variance. Thus, a more convincing example is called for here.

Fisher et al. (1995) discuss just such an example. Briefly, they consider two tasks: a double stimulation task like that run by Pashler and Johnston (1989) and referred to earlier in our discussion of exogenous processes; and a standard memory search task. To begin, consider the double stimulation task. Fisher et al. predict the response times at the three different SOAs of 50, 100, and $150 \mathrm{msec}$ and find that the younger adults take, respectively, 352 , 379 , and $415 \mathrm{msec}$ to respond to the second of two stimuli. To simulate general slowing, they assume that the durations of the latent processes in the older adults are slowed by a factor of two. Doing this, they predict that the older adults will take 683,704 , and $737 \mathrm{msec}$ at, respectively, the three SOAs above (note that the response times of the older adults are not a simple doubling of the response times of the younger adults, because of the presence of exogenous processes in double stimulation tasks). Now, consider the memory search task, which contains only endogenous processes. Imagine varying the number $u$ of items in the memory set and recording response time for the younger adults at each memory set size. Suppose that response times of 300,350 , and $400 \mathrm{msec}$ are recorded. Now increase the durations of the three latent processes by a factor of two. Again vary the number of items in the memory set and record the response time for the older adults at each memory set size. Given the model of memory search in Equation 15, it follows immediately that response times of 600,700 , and $800 \mathrm{msec}$ will be recorded. If older adults' response times in the double stimulation and memory search task (six conditions altogether) are now regressed on younger adults' response times in these two tasks, using a slowing factor common to all six conditions (where $\alpha$ is set to zero, since we are assuming that the molar model is a multiplicative one), the regression explains just $70 \%$ of the variance (i.e., the molar multiplicative model of general slowing explains just $70 \%$ of the variance). However, if the regression above is undertaken with separate slowing factors for the two tasks, the regression explains $91 \%$ of the variance (i.e., the molar multiplicative model of task-specific slowing explains fully $91 \%$ of the variance). Thus, the investigator might be tempted to reject the molar model of general slowing and associated latent model of common slowing. Yet, by construction, the latent model of common slowing explains $100 \%$ of the variance. Thus, as claimed above, it is necessary to test a latent model of common slowing even if a molar model of general slowing fits quite poorly (relatively).

\section{DISCUSSION}

The molar slowing function has served a central role in research on aging, dementia, depression, development, and intelligence. In particular, logically it has been assumed that if the molar slowing function is a general one, the latent slowing function is a common one. Similarly, statistically it has been assumed that if the fit of a general, linear molar slowing function is very good, the latent slowing function is most likely to be a common one. We have argued that both these inferences can lead, and indeed have led, to false positive decisions. Conversely, it has been assumed that if the molar slowing function is not a general one, the latent slowing function must be process-specific. Similarly, it has been assumed that if the fit of a general, linear molar slowing function is not very good, the latent slowing function is most likely processspecific. We have argued that both of these inferences can lead, and again have led, to false negative decisions.

Three issues remain to be addressed at this point. First, it is important to discuss exactly what actions are needed in order to avoid false positive and false negative decisions. Second, it is important to discuss how to use the latent modeling techniques to test a composite model of cognitive slowing where the speeds of individual processes are assumed to be determined by both general and specific factors. Finally, it is important to discuss, at least briefly, what needs to be done when the range of response times is longer than $2,000 \mathrm{msec}$ and something like an information loss model (Myerson et al., 1990) must be used to explain the results.

\section{Latent and Molar Models of Slowing}

Ideally, investigators could use the molar slowing function by itself to determine whether the latent slowing function was a common or a process-specific one, since the molar slowing function is relatively simple to evaluate. The question at this point is what additional tests are required, if any, in order to avoid the logical and statistical false positive and false negative decisions described above. Although the reader could easily infer from the above just what is required, it is perhaps most straightforward quickly to summarize the necessary actions.

First, consider false negative decisions. Logically, the molar slowing function is not by itself sufficient. To see this, recall that one set of conditions must be satisfied when there are no resource constraints (Theorem 1), a second set when there are resource constraints (Theorem 2), and still a third set when there are dependencies among the process durations (Corollary 1). Common to the set of constraints 
is the assumption that the processes are arranged in a PERT network. In order to determine that the cognitive network is indeed a PERT network, one needs at a minimum to undertake the tests proposed by Schweickert and Townsend (1989; see also Schweickert et al., 1993). Such tests, while they do not require a clearly formulated stochastic model of the response times (and the attendant assumptions about the distributions of the durations of the latent processes), do require a reasonably extensive factorial manipulation of the durations of the putative latent processes. Statistically, it is also not sufficient simply to examine the molar slowing function. Now, however, one needs actually to fit both latent models of common and process-specific slowing to the response time results to make sure that the poor fit of the molar model of general slowing is due to a failure of the common slowing hypothesis.

Next, consider false positive decisions. Logically, it seems relatively unlikely that one would falsely accept a molar model of general slowing, since the conditions in which one could do this are relatively infrequent. That is, relatively few iterative tasks are such as they are defined here (although see Hale et al., 1995, for a somewhat broader definition of iterative tasks). However, statistically, the studies analyzed here suggest that a complete analysis of the slowing of the latent processes will be required in order to avoid falsely accepting the hypothesis that all processes are slowed identically (proportionately the same in this context). The complete analysis requires, as above, a specification of a fully formulated stochastic model of response times.

In summary, it would appear that both logical and statistical considerations suggest the need for tests of a latent model, either tests of the structure (Schweickert \& Townsend, 1989) or tests of participants' actual behavior (Fisher \& Goldstein, 1983; Goldstein \& Fisher, 1991, 1992). These tests are relatively easy to perform as long as the model of processing has relatively few processes (Schweickert et al., 1993). Obviously, the difficulty increases as the number of processes increases.

\section{Composite Models of Cognitive Slowing}

Investigators have become interested in identifying which processes are slowed abnormally. Recently, Madden, Pierce, and Allen (1992) have described an approach with which one can identify such processes. Specifically, they suggest regressing the slower cohort's response times on the faster cohort's response times. A slowing function is determined. The raw score of each participant in the faster cohort is then transformed by the slowing function. Finally, an analysis of variance is used to analyze the transformed scores of the faster cohort and the raw (untransformed) scores of the slower cohort. If there is a significant interaction of age with any of the conditions, it is assumed that the processes that are manipulated in the relevant conditions are slowed significantly more than would be expected on the basis of the general or average slowing.

Clearly the logic of such a test works well when the underlying architecture is serial. A change in the duration of a given process by a set magnitude increases the overall response times by just the same magnitude. However, as we have shown, when the architecture is more complex (especially one with exogenous processes), there is no simple relation between the change in the duration of a given process and the overall response times. Thus, the method proposed by Madden, Pierce, and Allen (1992) may be compromised. However, the method proposed in this article can be used for just the purpose described by Madden et al. when the architecture is more complex. Specifically, since numerical estimates of the slowing functions are obtained, it is a simple matter to rank order these slowing functions from largest to smallest. However, the statistics that we have described cannot be used to determine whether a particular parameter is significantly larger than the average of the slowing functions. Additional statistical analyses are needed here as well.

\section{Latent Information Loss Slowing Function}

Above, the focus has been on molar and latent linear models of slowing. As noted there, such models are more than adequate when the response times lie in the range 0-2,000 msec. More complex models are needed when the response times are longer than $2,000 \mathrm{msec}$ (Cerella \& Hale, 1994; Cerella et al., 1980). We now want to shift focus to these more complex models and, in particular, to molar power models of general slowing and the related latent information loss models of common slowing (Hale et al., 1987; Myerson et al., 1990). Again, although the models will be taken from the aging literature (because that is where they are most fully developed), the conclusions reached from the study of the relation between the models apply much more broadly.

In its simplest form, a molar power model of general slowing has scaling parameters, $\zeta$ and $\delta$, that do not depend on the task (Hale et al., 1987):

$$
E\left[O_{i}\right]=\zeta E\left[Y_{i}\right]^{\delta} .
$$

Myerson et al. (1990) show that this molar power model of general slowing can be derived from what they call an information loss model. In order to make clear what we mean by such a model, we need briefly to discuss the assumptions made by Myerson et al. (1990) about the latent information loss model. First, they assume that the network that represents performance in each and every task for both the older and younger adults is a simple serial one. Second, they assume that for younger adults the expected duration $E\left[Z_{f}\right]$ of the $f$ th step (elementary cognitive unit) in the series is equal to the quantity $D /\left[I\left(1-q_{y}\right)^{f}\right]$, where $I$ is the proportion of information available at the outset of the task (relative to the minimal amount of information required to perform the task), where $D$ is a constant equal to the time required to perform the initial step ( $f=0$ at the outset) when the proportion $I$ of information is equal to 1 , and where $q_{y}$ is the proportion of information lost at each step. Similarly, they assume that for older adults the expected duration $E\left[\Phi_{f}\right]$ of the $f$ th step in the series is equal to the quantity $D /\left[I\left(1-q_{\mathrm{o}}\right)^{f}\right]$. Third, they assume that the proportion $q_{0}$ of information lost at each step by older adults is greater than the pro- 
portion $q_{y}$ of information lost at each step by younger adults, i.e., $q_{\mathrm{o}}>q_{\mathrm{y}}$. Let $\beta=q_{\mathrm{o}} / q_{\mathrm{y}}$.

Expressions for younger and older adults' response times can now be obtained for a latent information loss model when the processes are arranged in series. Let $t$ equal the total number of steps. Then, the expected time for younger adults to complete all $t$ steps if the latent information loss model governs behavior is simply

$$
E[Y]=E\left[Z_{0}+\ldots+Z_{t-1}\right]=\sum_{f=0}^{t-1} \frac{D}{I\left(1-q_{y}\right)^{f}} .
$$

Similarly, the expected time for older adults to complete all steps can be written as:

$$
E[O]=E\left[\Phi_{0}+\ldots+\Phi_{t-1}\right]=\sum_{f=0}^{t-1} \frac{D}{I\left(1-q_{0}\right)^{f}} .
$$

(Note that we could do away with the expectation operator, since all variables are constants; however, the operator will continue to be used here, since it keeps consistent the notation throughout.) It follows that the time $E\left[\Phi_{f}\right]$ that it takes the older adults to complete the $f$ th step can be written as a function of the step index $f$, a slowing constant $\left(1-q_{y}\right) /\left(1-\beta q_{y}\right)$, and the time $E\left[Z_{f}\right]$ that it takes the younger adults to complete this same step:

$$
E\left[\Phi_{f}\right]=\left(\frac{1-q_{y}}{1-\beta q_{y}}\right)^{f} E\left[Z_{f}\right] .
$$

To keep things simple, the latent slowing function in Equation 23 will be referred to as a latent information loss slowing function. In turn, the latent information loss slowing function will be referred to as a common slowing function, because the same constant is raised to a power across steps (or processes).

Typically, investigators will describe behavior in terms not of elemental steps but more general processes. In this case, one can imagine that process $x_{1}$ consists of $t_{1}$ steps, process $x_{2}$ consists of $t_{2}$ steps, and so on, where $t=t_{1}+$ $t_{2}+\ldots+t_{n}$. Then, for the younger adults we can write Equation 21 as follows:

$$
\begin{aligned}
E[Y]= & X_{1}+X_{2}+\ldots+X_{n} \\
= & \sum_{f=0}^{t_{1}-1} Z_{f}+\sum_{f=t_{1}}^{t_{1}+t_{2}-1} Z_{f}+\ldots+\sum_{f=t_{1}+\ldots+t_{n-1}}^{t_{1}+\ldots+t_{n}-1} Z_{f} \\
= & \sum_{f=0}^{t_{1}-1} \frac{D}{I\left(1-q_{\mathrm{y}}\right)^{f}}+\sum_{f=t_{1}}^{t_{1}+t_{2}-1} \frac{D}{I\left(1-q_{\mathrm{y}}\right)^{f}} \\
& +\ldots+\sum_{f=t_{1}+\ldots+t_{n-1}}^{t_{1}+\ldots+t_{n}-1} \frac{D}{I\left(1-q_{\mathrm{y}}\right)^{f}} .
\end{aligned}
$$

Equation 24 reduces immediately to Equation 21. Thus, it matters not for the younger adults whether one speaks in terms of elemental cognitive units or more general processes. Identical remarks apply to the older adults.

\section{Logical Analyses: False Negative Decisions}

Myerson et al. (1990) prove that if the latent slowing is governed by a common information loss function, the molar slowing is determined by a power function. They assume that the processes are arranged in series, that there are no resource constraints, and that the duration of one process is mutually independent of the durations of all other processes. Here, as above, it is of interest to know whether the same relation obtains when these assumptions are relaxed. In particular, it is important to avoid falsely rejecting the latent information loss model of common slowing solely on the basis of the knowledge that the molar power model of general slowing is not the correct one in situations in which the constraints are not so restrictive.

We want to consider relaxing the assumption that all processes are arranged in series. Specifically, assume that the latent processes are arranged in a more general PERT network (rather than only a straightforward serial network) and that the latent slowing function is a common information loss one. What is really rather surprising is that, given the satisfaction of some reasonably broad constraints, the molar power model of general slowing still describes performance when the processes are arranged in this more general network. The constraints include the same general assumptions as were made in considering latent multiplicative models: the processes in the network governing the performance of younger and older adults are endogenous, and identical networks describe the performance of younger and older adults in any given task. Additionally, it must be assumed that the duration of the elemental step size is a constant ( $D$ above). And, it must be assumed that the effect of changing the level of a factor is to increase (or decrease, as the case may be) the number of elemental steps in the affected processes. Then, if the latent slowing function is a common information loss one, it can be shown that the molar slowing function is a general power one (Appendix E).

\section{Logical Analyses: False Positive Decisions}

Above, we discussed the conditions that must obtain if one is to avoid falsely rejecting a latent information loss model of common slowing solely on the basis of the knowledge that the molar power model of general slowing is not the correct one. Now, we want to discuss the conditions that must obtain if one is to avoid falsely accepting the latent information loss model of common slowing solely on the basis of the knowledge that the molar power model of general slowing is the correct one. To undertake such a discussion, it is necessary to differentiate common and process-specific models. Although Myerson et al. (1990) do not indicate exactly how one should modify their latent information loss model of common slowing to create a latent information loss model of process-specific slowing, at least one modification appears reasonable and straightforward. Specifically, assume that the proportion of information lost for the older adults varies across at least two processes (also see Molenaar \& van der Molen, 1994). Thus, instead of just one proportion, $q_{0}$, governing the informa- 
tion loss across processes, there could be as many proportions as there were processes. Thus, in Equation 23, $\beta$ would be replaced by $\beta_{j}$ in a process-specific model. In keeping with the relation above between elemental steps and more general processes, it will be assumed that the information loss remains constant across steps within a process. At this point, the relation between the latent and molar slowing functions can be made clear. The development will proceed along much the same lines as above.

To begin, assume that the processes in each of $m$ tasks are arranged in a PERT network and assume that there are a total of $n$ unique processes along the $m$ critical paths in each task where $m \geq n$. To keep things simple, assume that there is only one step in each process so that $X_{j}=Z_{j}$ and $\Psi_{j}=\Phi_{j}$. Let $Z_{i j}$ represent the expected duration of the $j$ th process in the $i$ th task. Let $\Lambda$ in this context represent the $m \times n$ matrix $\left[Z_{i j}\right]$ of process durations. Then, if the columns of $\Lambda$ are linearly independent, the latent slowing function must be a common information loss slowing function (i.e., a process-specific information loss slowing function cannot be constructed which is consistent with the molar information loss model of general slowing). The proof is left as an exercise for the reader (in Theorem 3, Appendix C, simply replace the vector of common slowing coefficients with the coefficients in Equation 23 and replace the vector of process-specific slowing coefficients with the process-specific equivalents of the coefficients in Equation 23). Note that here it need not be assumed that the PERT network is serial in order to establish uniqueness. This contrasts with the requirement that the PERT network be serial in order to establish the uniqueness of a common multiplicative slowing function when the molar slowing function is a general, multiplicative one.

\section{Statistical Analyses}

Just as logically it is possible falsely to reject or accept the latent information loss model of common slowing, so too statistically it is possible falsely to accept or reject this model. A hypothetical situation in which one might falsely accept the latent information loss model of common slowing on the basis of the good fit of a molar power model of general slowing is well illustrated in Figure $2 \mathrm{~b}$. A situation in which one might falsely reject the latent information loss model of common slowing on the basis of the poor fit of a molar power model of general slowing can be constructed by using much the same logic as was used in the relevant earlier section.

\section{CONCLUSION}

It has been argued that the current procedures for identifying the effect of aging on the slowing of individual cognitive processes need to be expanded (and perhaps replaced). The likelihood that one might falsely reject or falsely accept the latent model of common slowing simply appears too great. Up until recently, the primary concern has been that the latent model of common slowing would wrongly be accepted (Fisk et al., 1992). Indeed, it was shown above that in a study widely cited as consistent with the latent model of common slowing (Salthouse \& Somberg, 1982), the latent model of processspecific slowing provides a better fit. And in a study (Fisk \& Rogers, 1991) also cited as consistent with a latent model of common slowing (Cerella, 1991), the latent model of process-specific slowing again provides a significantly better fit. In agreement with the finding above that a latent model of common slowing is inadequate, Hale et al. (1995) suggest that although the model may not work across all domains, it may be consistent with the results within particular domains. Given the recent tilt toward domainspecific models in the literature on aging, the primary concern at this moment is that the latent model of common slowing may be wrongly rejected if only the fit of this model (or the molar model of general slowing) within a domain is analyzed. The same concerns apply equally well to the attempt to understand the effect of the defining difference between other cohorts (e.g., levels of depression) on the slowing of cognitive processes.

The remedy suggested requires a more extensive series of tests than have generally been undertaken. In particular, the type of latent network must be identified (Schweickert \& Townsend, 1989). Predictions of response time must then be derived from the latent model and fit to the various conditions (Goldstein \& Fisher, 1992). Although the procedures are more elaborate, they have been used successfully to model behavior in a relatively broad range of complex tasks (Schweickert et al., 1993) and should prove adequate to the task at hand.

The tests have theoretical and practical benefits beyond those emphasized up to this point. Not only do the tests make it possible to differentiate reliably between latent models of common and process-specific slowing. But, additionally, in those cases in which the slowing is process specific, the tests make it possible to identify just which processes are slowed and by how much. Theoretically, one can then potentially identify which neural systems are most affected by the factor differentiating the cohorts under consideration (Bashore, 1993). And practically, one can then design systems for older adults which best mediate the effects of the processes most influenced by the differentiating factor, perhaps even optimizing the design (Fisher, 1993).

In summary, the preceding analyses suggest that the arguments used to support or to reject models of general slowing need to be reevaluated. Given the clear importance (both applied and theoretical) of understanding which processes, if any, in a given cohort are slowed, it seems imperative that one develop methods that make it possible to identify clearly the critical processes. It is hoped that the methods suggested here facilitate this identification.

\section{REFERENCES}

ANDERSON, J. R., \& BowER, G. H. (1974). Human associative memory. New York: Wiley.

BALL, K., \& OWSLEY, C. (1991). Identifying correlates of accident involvement for the older driver. Human Factors, 33, 583-595.

BASHORE, T. R. (1993). Differential effects of aging on the neurocognitive functions subserving speeded mental processing. In J. Cerella, 
J. Rybash, W. Hoyer, \& M. L. Commons (Eds.), Adult information processing: Limits on loss (pp. 37-76). New York: Academic Press. BirReN, J. E. (1965). Age changes in speed of behavior: Its central nature and physiological correlates. In A. T. Welford \& J. E. Birren (Eds.), Behavior, aging and the nervous system (pp. 191-216). Springfield, IL: C. C. Thomas.

Brinley, J. F. (1965). Cognitive sets, speed and accuracy of performance in the elderly. In A. T. Welford \& J. E. Birren (Eds.), Behavior, aging, and the nervous system (pp. 114-149). Springfield, IL: C. C. Thomas.

Cerella, J. (1985). Information processing rates in the elderly. Psychological Bulletin, 98, 67-83.

Cerella, J. (1990). Aging and information-processing rate. In J. E. Birren \& K. W. Schaie (Eds.), Handbook of the psychology of aging (3rd ed., pp. 201-221). San Diego: Academic Press.

Cerella, J. (1991). Age effects may be global not local: Comment on Fisk and Rogers (1991). Journal of Experimental Psychology: General, 120, 215-220.

Cerella, J., \& Hale, S. (1994). The rise and fall in informationprocessing rates over the life span. Acta Psychologica, 86, 109-197.

Cerella, J., Poon, L., \& Williams, D. M. (1980). Age and the complexity hypothesis. In L. W. Poon (Ed.), Aging in the 1980s: Psychological issues (pp. 332-340). Washington, DC: American Psychological Association.

Eriksen, C. W., Hamlin, R. M., \& Dayf, C. (1973). Aging adults and rate of memory scan. Bulletin of the Psychonomic Society, 1, 259-260.

FisHER, D. L. (1982). Limited channel models of automatic detection: Capacity and scanning in visual search. Psychological Review, 89, 662-692.

FisHeR, D. L. (1985). Network models of reaction time: The generalized OP diagram. In G. d'Ydewalle (Ed.), Cognition, information processing and motivation (Vol. 3, pp. 229-254). Amsterdam: North-Holland

FISHER, D. L. (1993). Optimal performance engineering. Human Factors, 35, 115-140.

FISHER, D. L. (in press). State models of skill acquisition: Optimizing the training of older adults. In W. A. Rogers, A. D. Fisk, \& N. Walker (Eds.), Aging and skilled performance: Advances in theory and applications.

Fisher, D. L., FISK, A. D., \& DufFy, S. A. (1995). Why latent models are needed to test hypotheses about the slowing of word and language processes in older adults. In P. Allen \& T. Bashore, Advances in psychology: Age differences in word and language processing (pp. 1-29) New York: Elsevier, North-Holland.

FISHER, D. L., \& GolDSTEIN, W. M. (1983). Stochastic PERT networks as models of cognition: Derivation of the mean, variance and distribution of reaction time using order-of-processing (OP) diagrams. Journal of Mathematical Psychology, 27, 121-151.

Fisher, D. L., Wisher, R, A., \& RANNEY, T. (1996). Static and dynamic training strategies: $\mathrm{A}$ framework for optimizing training strategies. Journal of Mathematical Psychology, 40, 30-47.

Fisk, A. D., \& Fisher, D. L. (1994). Brinley plots and theories of aging: The explicit, implicit and muddled debates. Journals of Gerontology, 49, P81-P89.

FisK, A. D., Fisher, D. L., \& Rogers, W. A. (1992). General slowing alone cannot explain age-related search effects: Reply to Cerella (1991). Journal of General Psychology, 121, 73-78.

FisK, A. D., \& RogERS, W. (1991). Toward an understanding of agerelated memory and visual search effects. Journal of Experimental Psychology: General, 120, 131-149.

GoldsteIn, W. M., \& FiSHER, D. L. (1991). Stochastic networks as models of cognition: Derivation of response time distributions using the order-of-processing method. Journal of Mathematical Psychology, 35, 214-241.

Goldstein, W. M., \& Fisher, D. L. (1992). Stochastic networks as models of cognition: Deriving predictions for resource-constrained mental processing. Journal of Mathematical Psychology, 36, 129-145.

GreEno, J., \& STEInER, T. (1964). Markovian processes with identifiable states: General considerations and application to all-or-none learning. Psychometrika, 29, 309-333.

HALE, S. (1990). A global developmental trend in cognitive processing speed in children. Child Development, 61, 653-663.

Hale, S., \& Myerson, J. (1992, April). Age-sensitivity and age- invariance in speeded information processing. Paper presented at the 4th annual Cognitive Aging Conference, Atlanta.

Hale, S., Myerson, J., Faust, M., \& Fristoe, N. (1995). Converging evidence for domain-specific slowing from multiple nonlexical tasks and multiple analytic methods. Journal of Gerontology: Psychological Sciences, 50B, P202-P211.

Hale, S., Myerson, J., \& Wagstaff, D. (1987). General slowing of nonverbal information processing: Evidence for a power law. Journal of Gerontology, 42, 131-136.

Hanowski, R. J., Bittner, A. C., JR., Knipling, R. R., Byrne, E. A., \& Parasuraman, R. (1995). Analysis of older-driver safety interventions: A human factors taxonomic approach. In Proceedings of the 1995 annual meetings of ITS America (Vol. 2, pp. 955-965). Washington, DC: ITS America.

HaRT, R. P., Kwentus, J. A., WADE, J. B., \& HAMER, R. M. (1987) Digit symbol performance in mild dementia and depression. Journal of Consulting \& Clinical Psychology, 55, 236-238.

HerTzOG, C. (1989). Influences of cognitive slowing on age differences in intelligence. Developmental Psychology, 25, 636-651.

HERTZOG, C. (1992). Aging, information processing speed, and intelligence. In K. W. Schaie (Ed.), Annual review of gerontology and geriatrics (Vol. 11, pp. 55-79). New York: Springer-Verlag.

HoHn, F. E. (1964). Elementary matrix algebra (2nd ed.). New York: Macmillan.

KAIL, R. (1991). Developmental change in speed of processing during childhood and adolescence. Psychological Bulletin, 109, 490-501.

KLIEGL, R. (1992, April). Becoming specific about age-related slowing: Person-based processing rates in simple and complex tasks. Paper presented at the 4th annual Cognitive Aging Conference, Atlanta.

Kleigl, R., Mayr, U., \& Krampe, R. T. (1994). Time-accuracy functions for determining process and person differences: An application to cognitive aging. Cognitive Psychology, 26, 134-164.

LAVER, G. D., \& BURKE, D. M. (1993). Why do semantic priming effects increase in old age? A meta-analysis. Psychology \& Aging, 8, 34-43

LiU, Y. (1996). Queueing network modeling of elementary mental processes. Psychological Review, 103, 116-136.

LORCH, R. F., JR., \& MYERS, J. L. (1990). Regression analyses of repeated measures data in cognitive research. Journal of Experimental Psychology: Learning, Memory, \& Cognition, 16, 149-157.

Madden, D. R., Nebes, R., \& Allen, P. A. (1992). Cognitive slowing in Alzheimer's disease as a function of task type and response type. Developmental Neuropsychology, 8, 459-471.

Madden, D. R., Pierce, T. W., \& Allen, P. A. (1992). Adult age differences in attentional allocation during memory search. Psychology \& Aging, 7, 594-601.

MAYR, U., \& KLEIGL, R. (1993). Sequential and coordinative complexity: Age-based processing limitations in figural transformations. Journal of Experimental Psychology: Learning, Memory, \& Cognition, 19, $1297-1320$.

McClelland, J. L., Rumelhart, D. E., \& The PDP Research Group (1986). Parallel distributed processing (Vol. 2). Cambridge, MA: MIT Press.

MILLER, J. O. (1993). A queue-series model for reaction time, with discrete-stage and continuous-flow models as special cases. Psychological Review, 100, 702-715.

Molenaar, P. C. M., \& van der Molen, M. W. (1994). On the discrimination between global and local trend hypotheses of life-span changes in processing speed. Acta Psychologica, 86, 273-293.

Myerson, J., Hale, S., Wagstaff, D., Poon, L. W., \& Smith, G. A. (1990). The information-loss model: A mathematical theory of agerelated cognitive slowing. Psychological Review, 97, 475-487.

Myerson, J., WaGstafF, D., \& Hale, S. (1994). Brinley plots, explained variance, and the analysis of differences in response latencies. Journals of Gerontology, 49, P72-P80.

Nebes, R. D., \& BRADY, C. B. (1992). Generalized cognitive slowing and severity of dementia in Alzheimer's disease: Implications for the interpretation of response-time data. Journal of Clinical \& Experimental Neuropsychology, 14, 317-326.

Nebes, R. D., \& MadDEN, D. J. (1988). Different patterns of cognitive slowing produced by Alzheimer's disease and normal aging. Psychology \& Aging, 3, 102-104. 
Neter, J., \& WaSSERMAN, W. (1974). Applied linear statistical models. Homewood, IL: R. D. Irwin.

NiEDEREHE, G. (1986). Depression and memory impairment in the aged. In L. W. Poon (Ed.), Handbook for clinical memory assessment of older adults (pp. 226-237). Washington, DC: American Psychological Association.

Pashler, H., \& Johnston, J. C. (1989). Chronometric evidence for central postponement in temporally overlapping tasks. Quarterly Journal of Experimental Psychology, 41A, 19-45.

PERFECT, T. J. (1994). What can Brinley plots tell us about cognitive aging? Journals of Gerontology, 49, P60-P64.

RousE, W. B. (1980). Systems engineering models of human-machine interaction. New York: North-Holland.

Rumelhart, D. E., McClelland, J. L., \& The PDP Research Group. (1986). Parallel distributed processing (Vol. I). Cambridge: MIT Press.

SALthouse, T. A. (1978). The role of memory in the age decline in digitsymbol substitution performance. Journal of Gerontology, 33, 232238.

SAlthouse, T. A. (1991). Mediation of adult age differences in cognition by reductions in working memory and speed of processing. Psychological Science, 2, 179-183.

SALTHOUSE, T. A. (1996). General and specific speed mediation of adult age differences in memory. Journals of Gerontology, 51 B, P30-P42.

Salthouse, T. A., Kausler, D. H., \& Saults, J. S. (1988). Utilization of path analytic procedures to investigate the role of processing resources in cognitive aging. Psychology \& Aging, 3, 158-166.

Salthouse, T. A., \& Mitchell, D. R. D. (1990). Effects of age and naturally occurring experience on spatial visualization performance. Developmental Psychology, 26, 845-854.

Salthouse, T. A., \& SomberG, B. L. (1982). Isolating the age deficit in speeded performance. Journal of Gerontology, 37, 59-63.

SCHAIE, K. W. (1989). Perceptual speed in adulthood: Cross-sectional and longitudinal studies. Psychology \& Aging, 4, 443-453.

SCHWEICKERT, R. (1978). A critical path generalization of the additive factor method: Analysis of a Stroop task. Journal of Mathematical Psychology, 18, 105-139.

SCHWEICKERT, R., FISHER, D. L., \& GoldSTEIN, W. M. (1993). General latent network theory: Structural and quantitative analysis of networks of cognitive processes. Unpublished manuscript available: Richard Schweickert, Department of Psychological Sciences, Purdue University,

SCHWEICKERT, R., \& TOWNSEND, J. T. (1989). A trichotomy: Interactions of factors prolonging sequential and concurrent mental processes in stochastic discrete mental (PERT) networks. Journal of Mathematical Psychology, 33, 328-347.

StaPlin, L., \& FisK, A. D. (1991). A cognitive engineering approach to improving signalized left-turn intersections. Human Factors, 33, 559572.

STERNBERG, S. (1969). Memory-scanning: Mental processes revealed by reaction time experiments. American Scientist, 57, 421-457.

StrANG, G. (1980). Linear algebra and its applications (2nd ed.). New York: Academic Press

Summala, H., \& Mikkola, T. (1994). Fatal accidents among car and truck drivers: Effects of fatigue, age, and alcohol consumption. Human Factors, 36, 315.326.

ToWNSEND, J. T. (1972). Some results concerning the identifiability of parallel and serial processes. British Journal of Mathematical \& Statistical Psychology, 25, 168-199.

TOWNSEND, J. T., \& ASHBY, F. G. (1983). The stochastic modeling of elementary psychological processes. Cambridge: Cambridge University Press.

VerNon, P. A., \& JENSEN, A. R. (1984). Individual and group differences in intelligence and speed of information processing. Personality \& Individual Differences, 5, 411-423.

\section{APPENDIX A \\ Latent Linear Slowing Function: Endogenous Processes}

THEOREM 1. For each task, suppose that $G_{\mathrm{o}}(V, E)$ and $G_{\mathrm{y}}(V, E)$ are identical PERT networks, that $x_{1}, \ldots, x_{n}$ are all endogenous, that $\Psi_{j}=B X_{j}$ for $j=1, \ldots, n$, and that $B, X_{1}, \ldots, X_{n}$ are $m u-$ tually independent. Then $E[O]=\beta E[Y]$ regardless of the task, where $E[B]=\beta$.

Proof. Briefly, let the paths in a PERT network be labeled 1, $\ldots, p$. Let $n(h)$ equal the number of processes on path $h$. Consider now just the younger adults. The sum $X_{h_{1}}+X_{h_{2}}+\ldots+$ $X_{h_{n(h)}}$ is equal to the duration of the $h$ th path, where $X_{h_{k}}$ is the duration of the $k$ th process on the $h$ th path. The expected response time for the younger adults is equal to the expected value of the maximum of the path durations:

$E[Y]=E\left[\max \left\{X_{1_{1}}+\ldots+X_{1_{n(1)}}, \ldots, X_{p_{1}}+\ldots+X_{p_{n(p)}}\right\}\right]$.

Consider next the older adults. The duration $\Psi_{j}$ of each process in the older adults' network is simply the product $B X_{j}$. Thus

$$
\begin{aligned}
E[O]= & E\left[\operatorname { m a x } \left\{B X_{1_{1}}+\ldots+B X_{1_{n(1)}}, \ldots, B X_{p_{1}}\right.\right. \\
& \left.\left.+\ldots+B X_{p_{n(p)}}\right\}\right] .
\end{aligned}
$$

The coefficient $B$ can be moved outside the maximum operator on the right-hand side of the equality in Equation 26, in which case it follows from Equation 25 that $E[O]=\beta E[Y]$. This completes the proof.

\section{APPENDIX B Latent Linear Slowing Function: Resource Constraints}

THEOREM 2. For each task, suppose that $G_{\mathrm{o}}(V, E)$ and $G_{\mathrm{y}}(V, E)$ are identical $P E R T$ networks, that $x_{1}, \ldots, x_{n}$ are all endogenous, that $P\left(\mathbf{W}_{\mathrm{o}} \leq \mathbf{w}\right)=P\left(\mathbf{W}_{\mathrm{y}} \leq \mathbf{w}\right)$, and that $v_{\mathrm{o}}(j, k, h)=\beta v_{\mathrm{y}}(j, k, h)$, $\beta \mathrm{s}<1$, for all processes $j$, states $k$, and paths $h$. Then $E[O]=$ $\beta^{-1} E[Y]$, regardless of the task.

Proof. Let $A_{h}$ equal the duration of the $h$ th path in the OP diagram for younger adults when it is taken. Let $P(h)$ equal the probability that path $h$ is taken. Then, if there are $p$ paths, the expected time $E[Y]$ to respond can be written as

$$
E[Y]=\sum_{h=1}^{p} E\left[A_{h}\right] P(h) .
$$

First, it will be shown that the probability that a particular path is taken in the OP diagram does not depend on the cohort of adults under consideration. Goldstein and Fisher (1992) derive an expression for the probability that path $h$ is followed. The probability depends on three events. In order to identify these events, it is necessary to introduce some additional notation. Let $X_{h_{k}}$ be the duration of the $k$ th process that is completed along path $h$. Define a column vector $\mathbf{X}_{h}$ as follows:

$$
\mathbf{X}_{h}=\left(X_{h_{1}}, \ldots, X_{h_{n(h)}}\right)^{\prime} .
$$

Without loss of generality, assume that the processes are labeled in the order in which they are completed along path $h$. Let $V_{h}$ be an $n(h) \times n(h)$ matrix, where the entry in row $j$ and column $k$ is the rate parameter $v_{y}(j, k, h)$ (note that this entry is set to zero if the process $j$ is not in the current set of the $k$ th state along path $h)$. Define $\mathbf{Y}$ to be the random vector $\left[\mathbf{I}-\mathbf{V}_{h}\left(\mathbf{V}_{h}^{\prime} \mathbf{V}_{h}\right)^{-1} \mathbf{V}_{h}^{\prime}\right] \mathbf{X}_{h}$ and $\mathbf{Z}$ to be the random vector $\left(\mathbf{V}_{h}^{\prime} \mathbf{V}_{h}\right)^{-1} \mathbf{V}_{h}^{\prime} \mathbf{X}_{h}$. The probability that a path $h$ which begins in state $s_{h_{1}}$ is followed can now be written as (Goldstein \& Fisher, 1992):

$$
\begin{aligned}
P(h)= & P\left[(\mathbf{Y}=0 \text { and } \mathbf{Z}>0) \mid\left(s_{h_{\mathrm{t}}} \text { is reached }\right)\right] \\
& \times P\left(s_{h_{1}} \text { is reached }\right) .
\end{aligned}
$$

For older adults, each rate parameter in $V_{h}$ needs to be multiplied by $\beta$. Thus, vectors $\mathbf{Y}$ and $\mathbf{Z}$ become: 


$$
\begin{aligned}
& \mathbf{Y}=\left[\mathbf{I}-\beta \mathbf{V}_{h}\left(\beta \mathbf{V}_{h}^{\prime} \beta \mathbf{V}_{h}\right)^{-1} \beta \mathbf{V}_{h}^{\prime}\right] \mathbf{X}_{h}, \\
& \mathbf{Z}=\left(\beta \mathbf{V}_{h}^{\prime} \beta \mathbf{V}_{h}\right)^{-1} \boldsymbol{\beta} \mathbf{V}_{h}^{\prime} \mathbf{X}_{h} .
\end{aligned}
$$

This leaves vector $\mathbf{Y}$ unchanged, since the $\beta$ s cancel. And, $\mathbf{Z} / \boldsymbol{\beta}>0$ if and only if $\mathbf{Z}>0$ since $\beta>0$. Thus, the probability that a given path $h$ is taken is the same for the younger and older adults.

Next it will be shown that the expected duration of a path for older adults is equal to $\beta^{-1} E\left[A_{h}\right]$. Specifically, consider the time $A_{h}$ that it takes younger adults to complete path $h$ in the OP diagram given that path $h$ is taken. Let $T_{h_{k}}$ be the duration of the $k$ th state along the $h$ th path for younger adults. By construction,

$$
A_{h}=T_{h_{1}}+\ldots T_{h_{n(h)}}
$$

Then, from Equation 14, we know that for the first state,

$$
T_{h_{1}}=\frac{W_{1}}{v_{\mathrm{y}}(1,1, h)}
$$

and for succeeding states,

$$
T_{h_{k}}=\frac{W_{k}-\sum_{k^{\prime}-1}^{k-1} v_{\mathrm{y}}\left(k, k^{\prime}, h\right) T_{h_{k^{\prime}}}}{v_{\mathrm{y}}(k, k, h)} .
$$

Since by assumption process $k$ is completed in state $k$ along path $h$, we can ignore rate parameters $v_{\mathrm{y}}\left(k, k^{\prime}, h\right), k^{\prime}>k$. Now, let $\Gamma_{h}$ be the duration of the $k$ th state along path $h$ for the older adults. Then, similar to Equations 32 and 33, we have for the older adults

$$
\Gamma_{h_{1}}=\frac{\mathrm{W}_{1}}{v_{\mathrm{o}}(1,1, h)},
$$

and for succeeding states,

$$
\Gamma_{h_{k}}=\frac{W_{k}-\sum_{k^{\prime}-1}^{k-1} v_{0}\left(k, k^{\prime}, h\right) \Gamma_{h_{k^{\prime}}}}{v_{\mathrm{o}}(k, k, h)} .
$$

By assumption, $v_{\mathrm{o}}\left(k, k^{\prime}, h\right)=\beta v_{\mathrm{y}}\left(k, k^{\prime}, h\right), k^{\prime}=1, \ldots, k-1$. Thus, Equation 34 can be rewritten as

$$
\Gamma_{h_{1}}=\frac{W_{1}}{v_{0}(1,1, h)}=\frac{W_{1}}{\beta v_{\mathrm{y}}(1,1, h)}=\beta^{-1} T_{h_{1}} .
$$

As the induction hypothesis, assume that for $k^{\prime}=2, \ldots, k-1$, $\Gamma_{h_{k^{\prime}}}=\beta^{-1} T_{h_{k^{\prime}}}$. Substituting $\Gamma_{h_{k^{\prime}}}=\beta^{-1} T_{h_{k^{\prime}}}$ into Equation 35 , we obtain

$$
\Gamma_{h_{k}}=\frac{W_{k}-\sum_{k^{\prime}-1}^{k-1} v_{0}(k, k, h) \beta^{-1} T_{h_{k^{\prime}}}}{v_{0}(k, k, h)}
$$

And substituting $v_{\mathrm{o}}\left(k, k^{\prime}, h\right)=\beta v_{\mathrm{y}}\left(k, k^{\prime}, h\right)$, we can rewrite Equation 37 as:

$$
\Gamma_{h_{k}}=\frac{\beta^{-1}\left[W_{k}-\sum_{k^{\prime}-1}^{k-1} v_{\mathrm{y}}(k, k, h) \beta^{-1} T_{h_{k^{\prime}}}\right]}{v_{\mathrm{y}}(k, k, h)},
$$

where the right-hand side is just $\beta^{-1} T_{h_{1}}$. Therefore, for older adults, the average time that it takes to complete a path is just $\beta^{-1} E\left[A_{h}\right]$. QED.

\section{APPENDIX C \\ Molar Linear Slowing Function: Identifiable Latent Models}

THEOREM 3. Suppose that $E\left[O_{i}\right]=\beta E\left[Y_{i}\right]$ for all $m$ tasks, $\Psi_{j}=\beta_{j} X_{j}$ for all $n$ unique processes $x_{1}, \ldots, x_{n}$, and that the rank of $\Lambda$ is equal to $n, m \geq n$. Further, for each task, suppose that $G_{\mathrm{o}}(V, E)$ and $G_{\mathrm{y}}(V, E)$ are identical serial networks and that the $n$ processes are all endogenous. Then only the latent model of common slowing is consistent with the molar model of general slowing; that is, the molar model of general slowing implies $\beta_{j}=\beta$ for all $j$.

Proof. It is well known that if the rank of an $m \times n$ matrix is equal to $n$, the columns are linearly independent (Hohn, 1964, p. 153). Furthermore, if the columns of a matrix are linearly independent, the inverse of the matrix $\Lambda^{\prime} \Lambda$ exists $\left(\Lambda^{\prime}\right.$ is the transpose of $\Lambda$; Strang, 1980, p. 109). Let $\beta=\left(\beta_{1}, \ldots, \beta_{n}\right)^{\prime}$ be a column vector where each component represents a slowing factor, assuming that at least two such factors are not identical (this vector is associated with the latent model of process-specific slowing). Let $\mathbf{B}=(b, \ldots, b)^{\prime}$ be a column vector where again each component represents a slowing factor, assuming now, however, that all such factors are identical (this vector is associated with the latent model of common slowing). Assume that by suitably selecting the values of the slowing factors, the latent models of process-specific and common slowing can be made to predict the same relation between the response times of older and younger adults as the molar model of general slowing. Then, since each task is a serial one with only endogenous processes, across tasks we must have

$$
\left(\begin{array}{cccc}
\mu_{11} & \mu_{12} & \cdots & \mu_{1 n} \\
\mu_{21} & \mu_{22} & \cdots & \mu_{2 n} \\
\vdots & \vdots & \cdots & \vdots \\
\mu_{m 1} & \mu_{m 2} & \cdots & \mu_{m n}
\end{array}\right) \cdot\left(\begin{array}{c}
\beta_{1} \\
\beta_{1} \\
\vdots \\
\beta_{n}
\end{array}\right)=\left(\begin{array}{cccc}
\mu_{11} & \mu_{12} & \cdots & \mu_{1 n} \\
\mu_{21} & \mu_{22} & \cdots & \mu_{2 n} \\
\vdots & \vdots & \ddots & \vdots \\
\mu_{m 1} & \mu_{m 2} & \cdots & \mu_{m n}
\end{array}\right) \cdot\left(\begin{array}{c}
b \\
b \\
\vdots \\
b
\end{array}\right)
$$

which can be written more simply as $\Lambda \beta=\Lambda \mathbf{B}$. Multiplying each side by the matrix product $\left(\Lambda^{\prime} \Lambda\right)^{-1} \Lambda^{\prime}$ leaves us with the equation $\beta=\mathbf{B}$, a contradiction, since we assumed at the outset that at least two of the slowing factors in the process-specific model were not identical to one another (in fact, all must be identical to one another). It follows that if the assumptions of Theorem 3 are satisfied, the latent model of common slowing, but not the latent model of process-specific slowing, is consistent with the molar model of general slowing.

\section{APPENDIX D \\ Molar Linear Slowing Function: Nonidentifiable Latent Models}

THEOREM 4. Suppose that $E\left[O_{i}\right]=\beta E\left[Y_{i}\right]$ for all $m$ tasks, that $\Psi_{j}=\beta_{j} X_{j}$ for all $n$ unique latent processes $x_{1}, \ldots, x_{n}$, and that the tasks are iterative. Further, for each task, suppose that $G_{0}(V, E)$ and $G_{\mathrm{y}}(V, E)$ are identical serial networks each with $n$ latent processes, and that the n processes are all endogenous. Then both a latent model of process-specific and a latent model of common slowing are consistent with the molar model of general slowing.

Proof. To see this, note that the fact that the serial networks are iterative means that the expected baseline duration $\mu_{j}$ of each process in task $i$ is lengthened by some proportion $\gamma_{j}$. If the latent model of process-specific slowing is not consistent with the latent model of common slowing, then, as above, we should 
find a contradiction when we solve the relevant set of simultaneous equations. In matrix form, we can write:

$$
\begin{aligned}
& \left(\begin{array}{cccc}
\gamma_{1} & 0 & \cdots & 0 \\
0 & \gamma_{2} & \cdots & 0 \\
\vdots & \vdots & \ddots & \vdots \\
0 & 0 & \cdots & \gamma_{n}
\end{array}\right) \cdot\left(\begin{array}{cccc}
\mu_{1} & \mu_{2} & \cdots & \mu_{n} \\
\mu_{1} & \mu_{2} & \cdots & \mu_{n} \\
\vdots & \vdots & \cdots & \vdots \\
\mu_{1} & \mu_{2} & \cdots & \mu_{n}
\end{array}\right) \cdot\left(\begin{array}{c}
\beta_{1} \\
\beta_{2} \\
\vdots \\
\beta_{n}
\end{array}\right) \\
= & \left(\begin{array}{cccc}
\gamma_{1} & 0 & \cdots & 0 \\
0 & \gamma^{2} & \cdots & 0 \\
\vdots & \vdots & \ddots & \vdots \\
0 & 0 & \cdots & \gamma_{n}
\end{array}\right) \cdot\left(\begin{array}{cccc}
\mu_{1} & \mu_{2} & \cdots & \mu_{n} \\
\mu_{1} & \mu_{2} & \cdots & \mu_{n} \\
\vdots & \vdots & \ddots & \vdots \\
\mu_{1} & \mu_{2} & \cdots & \mu_{n}
\end{array}\right) \cdot\left(\begin{array}{c}
b \\
b \\
\vdots \\
b
\end{array}\right) .
\end{aligned}
$$

Multiplying through by the inverse of the left-hand matrix (which will exist as long as $\gamma_{i}$ does not equal zero for some $i$ ), we can solve for $b$ in any equation, since all are identical:

$$
\beta_{1} \mu_{1}+\beta_{2} \mu_{2}+\ldots+\beta_{n} \mu_{n}=\beta\left(\mu_{1}+\mu_{2}+\ldots+\mu_{n}\right) \text {. }
$$

Thus, the latent model of process-specific slowing and the latent model of common slowing can both be made consistent with a molar model of general slowing. QED.

\section{APPENDIX E \\ Latent Information Loss Slowing Function}

Assume that each process $x_{j}$ in a PERT network is composed of a series of $t_{j}$ steps, $j=1, \ldots, n$. Given that the duration of each process in the PERT network is a constant, as long as the duration of each path through the network is unique, there will be one and only one critical path. Let $h^{*}$ represent the critical path in the network governing the behavior of younger adults. Without loss of generality, label the processes on the critical path $x_{1}, x_{2}, \ldots, x_{n}$ where $n^{*} \leq n$. Let $t^{*}$ equal the total number of steps along the critical path $h^{*}$, so that $t^{*}=t_{1}+t_{2}+\ldots+t_{n^{*}}$. Then,

THEOREM 5. For each task, suppose that $G_{0}(V, E)$ and $G_{\mathrm{y}}(V, E)$ are identical PERT networks, that $x_{1}, \ldots, x_{n^{*}}$ are all endogenous processes along the critical path, that

$$
\begin{aligned}
& X_{j}=\sum_{f=t_{1}+\ldots+t_{j-1}}^{t_{1}+\ldots+t_{j}-1} \frac{D}{I\left(1-q_{\mathrm{y}}\right)^{f}}, \\
& \Psi_{j}=\sum_{f=t_{1}+\ldots+t_{j-1}}^{t_{1}+\ldots+t_{j}-1} \frac{D}{I\left(1-q_{\mathrm{o}}\right)^{f}}, j \leq n^{*},
\end{aligned}
$$

that $D$ is a constant, and that the effect of changing the level of a factor that influences a given process on the critical path is to increase (or decrease) the number of steps in the process by the same amount for both the older and younger adults. Then $E[O]=\zeta E[Y]^{\delta}$, regardless of the task.
Proof. For younger adults, the time $A_{h^{*}}$ that it takes to complete critical path $h^{*}$ is given by the sum (see Equation 24):

$$
\begin{aligned}
E[Y]= & X_{1}+X_{2}+\ldots+X_{n^{*}} \\
= & \sum_{f=0}^{t_{1}-1} \frac{D}{I\left(1-q_{\mathrm{y}}\right)^{f}}+\sum_{f=t_{1}}^{t_{1}+t_{2}-1} \frac{D}{I\left(1-q_{\mathrm{y}}\right)^{f}} \\
& +\ldots+\sum_{f=t_{1}+\ldots+t_{n^{*}-1}}^{t_{1}+\ldots+t_{n^{*}-1}} \frac{D}{I\left(1-q_{\mathrm{y}}\right)^{f}}
\end{aligned}
$$

Now we assumed that the duration of path $h^{*}$ is longest in the network for younger adults. This implies that the duration of the path $h^{*}$ in the network for older adults is also the longest, since the total number of steps in each process does not change as a function of age.

The result is that we can write the response times for the older and younger adults as a function of the number of steps in the processes on the longest path-say, $h^{*}$. Specifically,

$$
\begin{aligned}
& E[O]=\sum_{f=0}^{t *-1} \frac{D}{I\left(1-q_{\mathrm{o}}\right)^{f}}, \\
& E[Y]=\sum_{f=0}^{t-1} \frac{D}{I\left(1-q_{\mathrm{y}}\right)^{f}}
\end{aligned}
$$

It follows immediately from Myerson et al. (1990) that the response times for the older adults can be written as a power function of the response times for younger adults which does not depend on the upper bound of summation; that is, as $t^{*}$ is varied, the relation between $E[O]$ and $E[Y]$ is well approximated by the power function $E[O]=\zeta E[Y]^{\delta}$.

Now, suppose that a second task is considered, one in which the number of steps in critical process $x_{j}$ has been increased by some constant number-say $a$-for both the older and younger adults. For example, one might manipulate a factor that influenced the clarity of a stimulus (which in turn would increase or decrease the number of steps in the associated critical process). Then, the number of steps on the critical path is given by the $\operatorname{sum} t^{*}+a$. Equation 44 can be rewritten, using this new upper bound of summation for both the older and younger adults. But, since the power function relating older and younger adults' response times does not depend on the size of the upper bound of summation, the power function in this new task is identical to the power function in the baseline task. QED.

(Manuscript received January 20, 1996; revision accepted for publication August 14, 1996.) 